NBER WORKING PAPER SERIES

\title{
HOW SHOULD POLICY RESPONSES TO THE COVID-19 PANDEMIC DIFFER IN THE DEVELOPING WORLD?
}

\author{
Titan Alon \\ Minki Kim \\ David Lagakos \\ Mitchell VanVuren \\ Working Paper 27273 \\ http://www.nber.org/papers/w27273
NATIONAL BUREAU OF ECONOMIC RESEARCH
1050 Massachusetts Avenue
Cambridge, MA 02138
May 2020

For helpful comments we thank Gaby Cugat, James Dzansi, Norman Loayza, Valerie Ramey, Nii Sowa, Ernesto Stein, Henry Telli and seminar/conference participants at Exeter, Geneva, the IADB, UCSD and the World Bank. The views expressed herein are those of the authors and do not necessarily reflect the views of the National Bureau of Economic Research.

NBER working papers are circulated for discussion and comment purposes. They have not been peerreviewed or been subject to the review by the NBER Board of Directors that accompanies official NBER publications.

(C) 2020 by Titan Alon, Minki Kim, David Lagakos, and Mitchell VanVuren. All rights reserved. Short sections of text, not to exceed two paragraphs, may be quoted without explicit permission provided that full credit, including (C) notice, is given to the source. 
How Should Policy Responses to the COVID-19 Pandemic Differ in the Developing World? Titan Alon, Minki Kim, David Lagakos, and Mitchell VanVuren

NBER Working Paper No. 27273

May 2020, Revised August 2020

JEL No. E2,E6,I15,I28,O17,O41

\begin{abstract}
$\underline{\text { ABSTRACT }}$
This paper quantitatively analyzes how policy responses to the COVID-19 pandemic should differ in developing countries. To do so we build an incomplete-markets macroeconomic model with heterogeneous agents and epidemiological dynamics that features several of the key distinctions between advanced and developing economies germane to the pandemic. We focus in particular on differences in: age structure, fiscal capacity, healthcare capacity, informality, and the frequency of contacts between individuals at home, work, school and other locations. The model predicts that blanket lockdowns are less effective in developing countries, saving fewer lives per unit of lost GDP. In contrast, age-specific policies are even more effective, since they focus scarce public funds on shielding the smaller population of older individuals. School closures are also more effective at saving lives in developing countries, providing a greater reduction in secondary transmissions between children and older adults at home.
\end{abstract}

Titan Alon

University of California San Diego

Department of Economics

9500 Gilman Drive \#0508

La Jolla, CA 92093

titanalon@gmail.com

Minki Kim

University of California San Diego

Department of Economics

9500 Gilman Drive \#0508

La Jolla, CA 92093-0508

minkikim@ucsd.edu
David Lagakos

Department of Economics

Boston University

270 Bay State Road

Boston, MA 02215

and NBER

lagakos@bu.edu

Mitchell VanVuren

University of California San Diego

Department of Economics

9500 Gilman Drive \#0508

La Jolla, CA 92093-0508

mvanvure@ucsd.edu 


\section{Introduction}

Governments in advanced economies have responded to the COVID-19 pandemic with dramatic and unprecedented policy responses. Huge swaths of the economy have been ordered shut down and millions of workers required to stay home indefinitely. To cushion against the loss of income, governments have sent direct transfers to workers affected by the lockdowns, in addition to making regular social insurance payments. The total costs of these and other transfers has passed 10 percent of GDP in a number of advanced countries.

As COVID-19 made its way to less-developed countries, policy makers there largely followed suit with similarly sweeping lockdowns. Yet it quickly became clear that policy responses in the developing world could not just mimic those of the west (Barnett-Howell and Mobarak, 2020; Díez et al., 2020; Kim and Loayza, 2020; Pagés et al., 2020). Policy would instead have to be tailored to the dramatically different economic and demographic landscape that characterizes most low-income countries. Governments in these countries lack the fiscal capacity to make substantial transfers to major segments of the population for long periods. High levels of poverty mean that many households are effectively living hand-to-mouth, rendering prolonged lockdowns economically infeasible. Larger informal sectors make enforcing lockdowns more difficult. The potential health consequences of the pandemic are also distinct, given the younger populations but less developed healthcare systems in low-income countries. In addition, more crowded, intergenerational dwellings leads to more frequent transmissions via household contacts that are not readily controlled by lockdowns.

This paper provides a quantitative analysis of how policy responses to the pandemic in developing countries should differ from those of the west, given their sharply different characteristics. We focus on broad aggregate economic policy, meaning over the extent and duration of lockdowns and transfers, including whether either should be age-specific or accompanied by auxiliary measures, such as school closures. We also illustrate how the fiscal and healthcare capacity constraints interact with informality, intergenerational cohabitation, and younger demographics to inform optimal policy responses relative to advanced economies.

To do so, we follow the newly emerged literature on the macroeconomics of pandemics by combining a workhorse macro model with a variant of the SIR model standard in epidemiology. In particular, we build a heterogenous agent framework with uninsurable income shocks, as in Kaplan, Moll, and Violante (2020), and health risks that vary by age, as in Glover, Heathcote, Krueger, and Ríos-Rull (2020). In addition, our model features fiscal capacity constraints that reduce the ability of governments to tax and transfer resources across households (e.g. Besley and Persson, 2009; Jensen, 2019). It adds an informal sector as in Ulyssea (2018) and a large 
development literature, and worker sorting on skill as in Roy (1951) and many recent multisector models of structural change.

We capture epidemiological dynamics in the model using a variant of the SICR model that is standard in the epidemiology literature, combined with economic choices that govern the disease path endogenously, as in the macroeconomic literature on disease transmission (e.g. Greenwood, Kircher, Santos, and Tertilt, 2019; Eichenbaum, Rebelo, and Trabandt, 2020; Alvarez, Argente, and Lippi, 2020; Chang and Velasco, 2020, and many more). In contrast to most existing economic studies, our epidemiological model incorporates contact matrices measuring the number of contacts between individuals of different ages in different locations. In this vein, our approach is similar to that of Baqaee, Farhi, Mina, and Stock (2020), though we extend this approach beyond the United States by drawing on contact matrices for a wide range of countries. The contact matrices are important for capturing differences in social mixing and cohabitation patterns across countries, and crucial for understanding the effect of any targeted policy that does not apply to the entire population in all locations

We parameterize the model to match the pre-pandemic stationary distribution of a representative advanced economy, calibrated to match characteristics of countries in the top quartile of the world income distribution. We then compute the model's equilibrium response to the COVID-19 pandemic as a surprise "MIT shock" where a small exogenous fraction of the population becomes infected with the coronavirus. The model's infection and fatality rates are chosen to match the widely cited epidemiological studies of Ferguson et al. (2020) and Glynn (2020). The disease transmission rates by age are chosen to match the trajectory of cumulative deaths in the US from April through July 2020 and a relative infection rate of children consistent with the large-scale seroprevalence study in Spain by Pollán et al. (2020).

We then do the same for an alternative calibration of the model taken to match a representative developing economy, matching averages of the lowest-income quartile of countries. We match in particular the substantially lower population share of the old, the lower fiscal and healthcare capacity, the larger informal sector, and differing contact matrices at home, work, school and at other locations. We validate the model's fit on several dimensions and, in particular, show that it can replicate the steeper trajectory of fatalities in Brazil, which we do not target directly.

We then simulate the effects of various types of lockdowns in the advanced and developing economies to compare their impacts on GDP, fatalities, and consumption-equivalent welfare. The model predicts that blanket lockdowns are not as effective in developing countries, saving substantially fewer lives per unit of GDP lost. In contrast, age-dependent policies, like those studied by Bairoliya and Imrohoroglu (2020), and Acemoglu, Chernozhukov, Werning, and Whinston (2020) for advanced economies, are even more potent in a developing world context. 
In particular, an age-specific lockdown with closed schools saves five times as many lives per unit of GDP lost as in advanced economies, and nearly thirteen times as many lives as under blanket lockdowns in developing economies. The reason is that age-specific policies allow governments to isolate only those with the highest fatality risk, and to provide them with larger transfers than under blanket lockdowns. This is particularly attractive in developing countries, since older individuals constitute a smaller share of the population and limited fiscal capacity makes sustaining large economy-wide transfers infeasible.

Finally, our model predicts that school closures are much more effective at saving lives in developing economies than in advanced ones. Furthermore, while school closures predominantly affect children, nearly all the additional lives saved are amongst adults and the elderly. The main drivers of these results are the more crowded schools and greater prevalence of intergenerational cohabitation in developing economies, which together substantially increase the risk that children become infected at school and transmit the virus to older adults at home.

Our work is one of several in a growing literature on the macroeconomic consequences of the COVID-19 pandemic for the developing world. von Carnap, Almås, Bold, Ghisolfi, and Sandefur (2020) use a representative agent framework similar to Eichenbaum et al. (2020) to analyze the impact of lockdowns in Uganda, finding that lockdowns are less effective than in the west, as we do. Alfaro, Becerra, and Eslava (2020) take an empirical approach to understanding the effects of COVID-19 in Colombia, emphasizing the importance of informality for shaping the aggregate consequences of lockdowns. There are many aspects of developing economies that we have not modeled in this paper. Most prominent is the absence of open-economy considerations, which are taken up by Çakmakh, Demiralp, Ș. Kalemli-Özcan, Yesiltas, and Yildirim (2020) and Arellano, Bai, and Mihalache (2020), among others. We also abstract from differences in the prevalence of comorbities, such as diabetes and cardiovascular disease, which may affect fatality rates. Differential testing and tracing policies, as in Berger, Herkenhoff, and Mongey (2020), and imperfect information, as in Baker, Bloom, Davis, and Terry (2020), are also absent, though clearly relevant for understanding the effects of the pandemic.

\section{Motivating Facts for Differing Policy Responses in Developing Countries}

We begin by summarizing five important demographic and economic characteristics that differ across countries in ways that we view as essential for understanding how policy responses to COVID-19 should differ in developing economies. These are: (i) the much younger populations in developing countries, (ii) their lower fiscal capacity, (iii) their more widespread informality, (iv) their lower healthcare capacity, and (v) more frequent person-to-person contacts and a greater prevalence of intergenerational cohabitation. To be sure, all of these patterns are 
already known, and indeed, they have been emphasized in other studies of COVID-19 in the developing world (Kim and Loayza, 2020; Pagés et al., 2020; Díez et al., 2020). The goal of this section is to present these patterns in a systematic way and briefly highlight their relevance for the effects of the pandemic, which will help motivate the model and quantitative analysis that follow.

\subsection{Younger Populations}

It's well known by now that COVID-19 poses dramatically greater health risks to older individuals, in particular those over the age of 65 (Ferguson et al., 2020; Glynn, 2020). Early centers of infection in the west, such as Italy, experienced health impacts concentrated on those in this older age range, with particularly severe fatality rates for those in their $80 \mathrm{~s}$ and $90 \mathrm{~s}$. At the same time, the number of deaths linked to COVID-19 for those under 20 has been negligible, though certainly not zero.

A basic demographic difference between advanced and developing economies is that populations are far younger in the developing world. Since fatality rates from COVID-19 are very low for young individuals but rise sharply with age, these demographic differences suggest much smaller populations of vulnerable individuals in the developing world. One can see these demographic differences starkly when looking at cross-country data on the median age. Figure 1 plots the median age against GDP per capita in a set of 158 countries using data from UN Population Division and Penn World Tables. Data from the UN Population Division show that countries in the bottom quartile of the world income distribution have a median age of 19.1 years. Nigeria, Africa's most populous country, has a median age of 17.9, while countries like Angola and the Democratic Republic of the Congo have median ages of just 16.4 and 16.8 years old. By contrast richer countries like Italy, the United Kingdom and France have median ages of 45.9, 40.2 and 41.2, respectively. 
Figure 1: Median Age of the Population

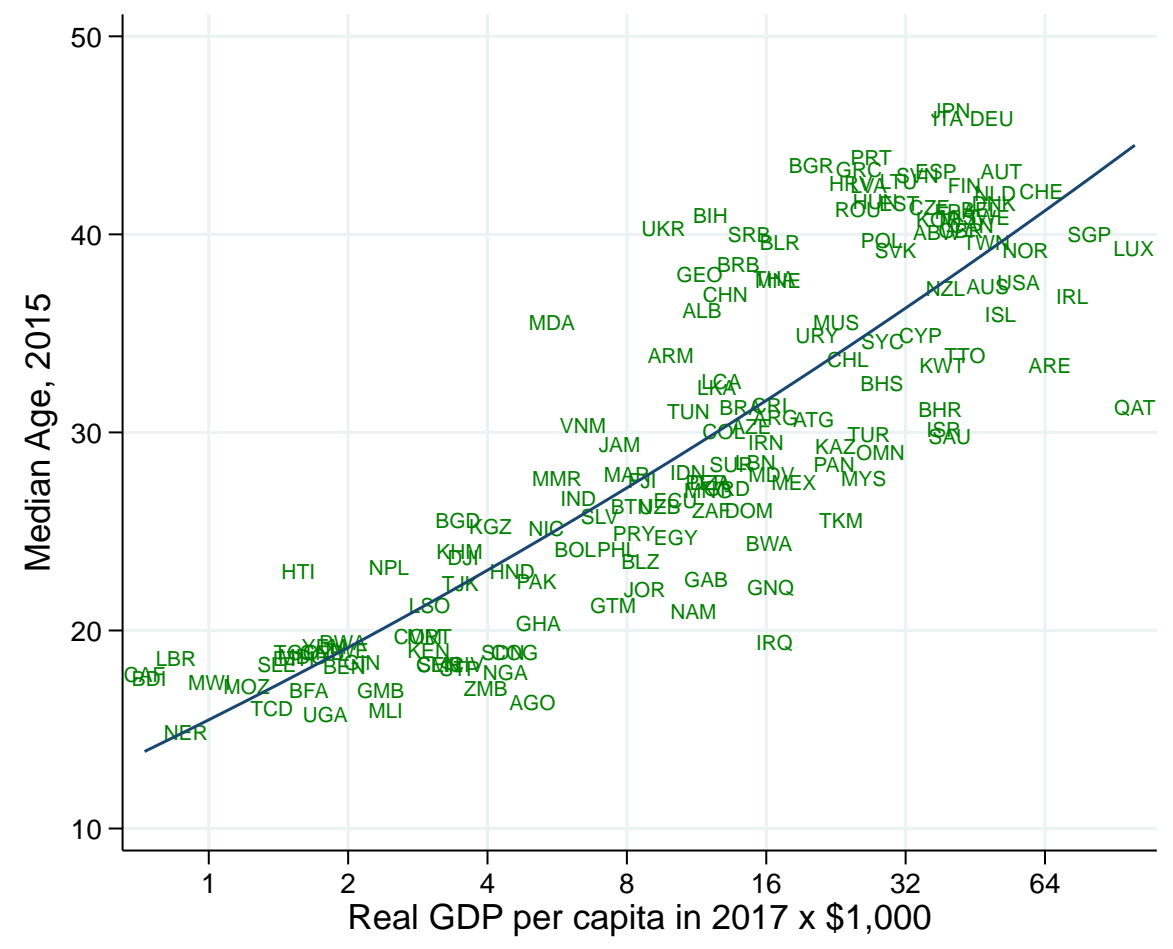

Note: This figure plots the median age in 2015 across 158 countries by GDP per capita. GDP per capita is expressed at PPP and taken from Penn World Table 9.1 (Feenstra et al., 2015). Median age data is from the UN Population Division.

Another statistic indicative of the much smaller vulnerable population in the developing world is the cross-country data on the population above 65. Figure A.1 plots the fraction of the population that is above 65 against GDP per capita in a set of 162 countries using data from the World Bank and the Penn World Tables. In the world's poorest countries the fraction of the population that is above age 65 is negligible, with an average of around 3 percent for countries in the bottom quartile of the world income distribution. The older population is much larger as a fraction of the total in richer economies, and reaches around one quarter of the population in Japan. Among countries in the topic quartile of the world, the average is about 15 percent of the population being above age 65 .

It is hard to look at statistics like these and not see how different the impacts of COVID-19 will be in less developed countries. Concretely, while almost everything about COVID-19 suggests a more severe impact in less-developed countries, the far younger demographic is clearly in their favor. 
Figure 2: Fiscal Capacity, Proxied by the Ratio of Taxes to GDP

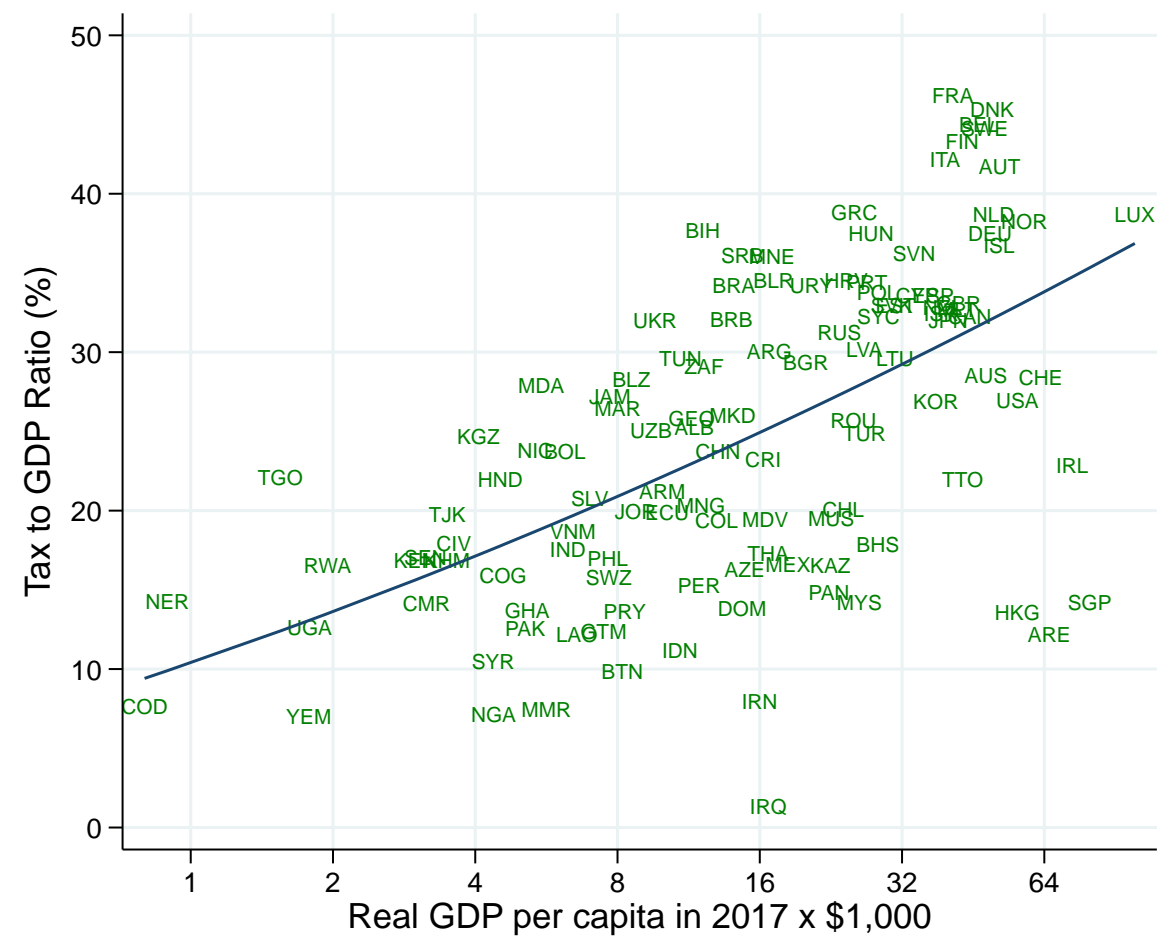

Note: This figure plots the ratio of taxation to GDP ratio across 116 countries. Tax revenues include social contributions. GDP per capita is expressed at PPP and taken from Penn World Table 9.1 (Feenstra et al., 2015). Tax revenue data is from the ICTD / UNU-WIDER Government Revenue Dataset 2019 (ICTD/UNUWIDER, 2019).

\subsection{Lower Fiscal Capacity}

Developed nations take for granted their government's ability to raise tax revenues and use the proceeds to provide public goods and make transfers. This fiscal capacity is not shared by the public sectors in developing countries, as a long literature has emphasized (see Besley and Persson, 2013, for an overview). This literature has emphasized how developing nations generally lack the ability to monitor and enforce tax payments from its citizens, and have less efficient revenue authorities than do richer countries.

Figure 2 plots total tax revenues as a fraction of GDP taken from the ICTD Government Revenue Dataset against GDP per capita. The nearly linear positive relationship between taxation relative to GDP and income per capita highlights the much lower role that taxation plays in less developed economies. Similar patterns have been observed in the time series for countries as they develop and increase rates of taxation, particularly on labor income (Besley and Persson, 2013; Jensen, 2019). 
The lower fiscal capacity in poorer countries is relevant for studies of the pandemic for several reasons. Most importantly, it limits the ability of governments to institute large-scale income replacement programs for furloughed workers during lengthy lockdowns or in response to widespread business closures. Quantitively, these limits appear to be substantial. Figure A.2 shows that share of GDP spent on public programs combatting the pandemic range from 2 percent in developing countries to 7 percent in advanced ones. Not receiving any payments is a clear disincentive for citizens to comply with a lockdown, especially for those that have little savings to fall back on. In addition, the inability to raise taxes effectively limits governments' ability to borrow, which further reduces their ability to make payments to furloughed workers.

\subsection{Larger Informal Sectors}

A large share of employment in developing countries is concentrated in informal production activities. By definition, such markets are beyond the purview of the state to tax or regulate, and make law enforcement difficult. Lockdown policies, which call for citizens not to leave home for work or for other public interactions, are difficult to enforce anywhere. Clearly, enforcing lockdowns is even harder in an environment with only a minority of the workforce employed at formal businesses.

Figure 3 plots the size of the informal sector as a fraction of non-agricultural employment, as measured by the International Labor Organization (ILO), against income per capita. It shows a sharp decline in informality rates in non-agricultural activities with GDP per capita. The countries with the lowest income have informality rates above 80 percent in most cases. The richest countries in this ILO database have informality rates below half in most cases, though this figure excludes most of the richest countries in the world, which undoubtedly have even lower rates of informality.

An obvious way in which informality ties the hands of governments in low-income economies is that it reduces their ability to collect additional taxes and make transfers to those in lockdown. The widespread informality and limited fiscal capacity are of course very closely linked, with each reinforcing the other. During the pandemic, any attempts to keep households in lockdown may increase the size of the informal sector, which hurts attempts to control disease or raise new revenues.

A related feature of the informal sector relevant for policy responses to the pandemic is the concentration of low-skilled jobs there. To the extent that lockdown policy forces desperate workers that have run down their savings to enter the informal sector, these workers can be expected to perform marginal tasks that do not generate much income. For workers already in marginal tasks in the formal sector, this may not represent much of a change. But for those in 
Figure 3: Size of the Informal Sector

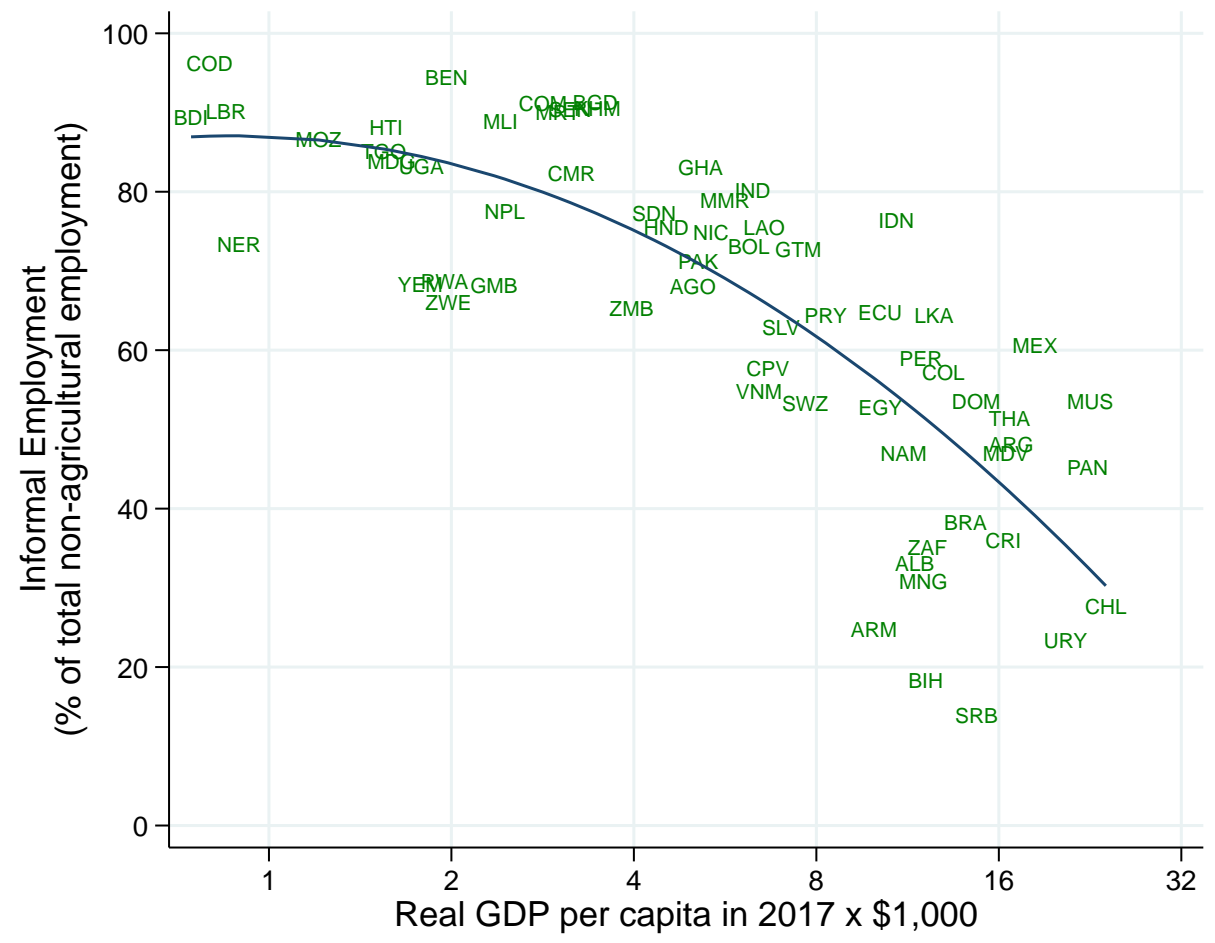

Note: This figure plots the employment in the informal economy, measured by the ILO, as a percentage of total non-agricultural employment, in 63 countries. The informal economy is defined as in the text. GDP per capita is expressed at PPP and is taken from the Penn World Table 9.1 (Feenstra et al., 2015). Informality data is from the ILOSTAT database.

more skilled jobs to begin with, having to work in low-skilled informal activities would represent a substantial decline in household income and therefore consumption. If enough workers become desperate and enter the informal sector, this could reduce aggregate productivity and further shrink the government's tax base.

\subsection{Lower Healthcare Capacity}

Developing countries typically have substantially less ability to control disease than do richer countries. Sanitation and hygiene are more of an issue given the lack of widespread piped water and functioning sewage systems. Health infrastructure, especially hospital and health clinic capacity, is also less developed. For mild cases of COVID-19 infections, this may make little differences, as bed rest is likely to suffice in these mild cases. However, for critical cases, the lack of intensive-care capacity is a clear disadvantage for developing countries in their attempts to save lives during the pandemic.

Figure 4 plots the number of hospital beds per 10,000 people, as reported by the World Health 
Figure 4: Healthcare Capacity, Proxied by Hospital Beds per 10,000 People

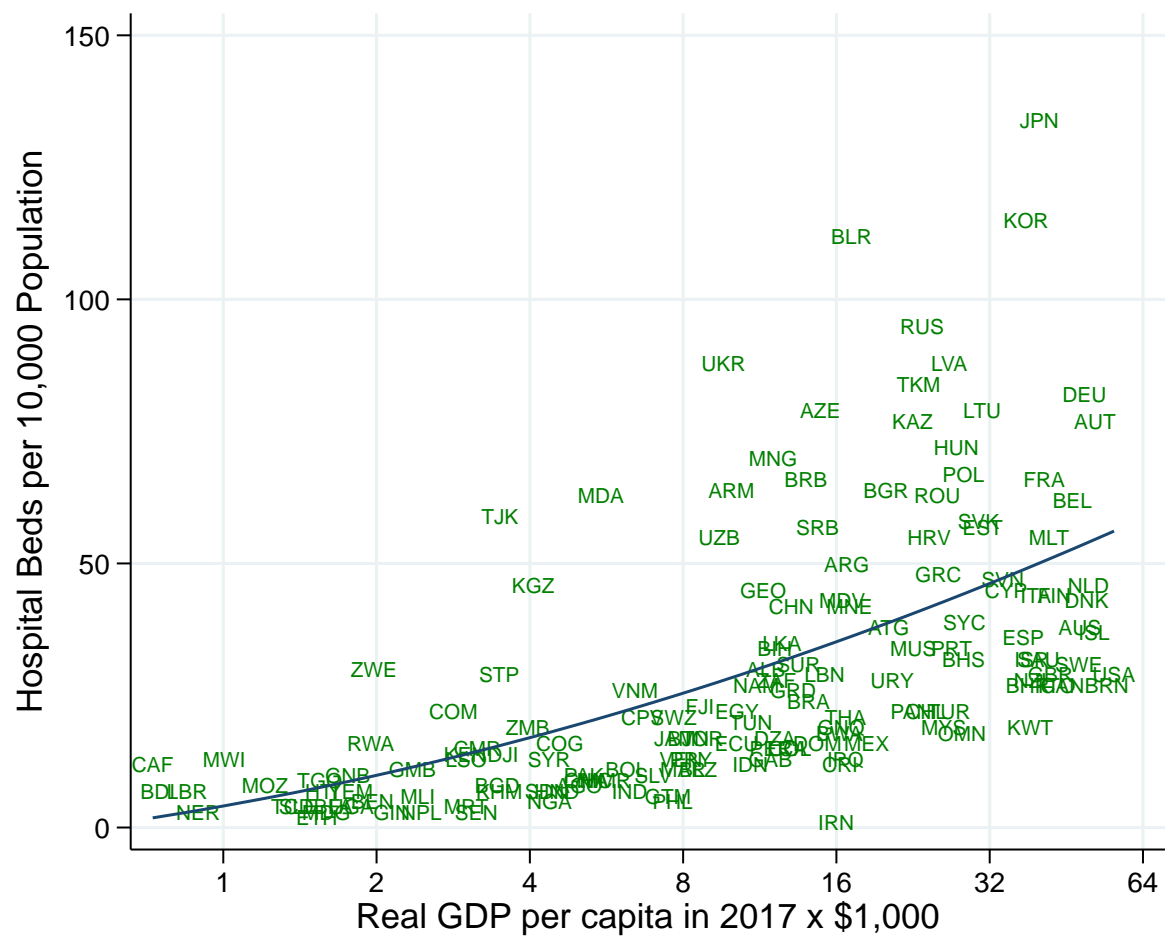

Note: This figure plots the number of hospital beds available per 10,000 inhabitants in 153 countries. GDP per capita is at PPP and taken from the Penn World Table 9.1 (Feenstra et al., 2015). The hospital bed data are from the World Health Organization's Global Health Observatory.

Organization (WHO), against GDP per capita. The number of hospital beds is an imperfect measure of hospital capacity for many reasons, most importantly because it is not a bed per se that helps critical patients recover from COVID-19 but trained doctors, equipment like ventilators, and appropriate pharmaceuticals. Still, for lack of more comprehensive cross-country data, we take hospital beds as a proxy for medical care capacity.

By this metric there are stark differences in healthcare capacity across countries. Richer countries, which have quite some range amongst themselves, average around 49 hospital beds per 10,000 people. Countries like Japan and Korea have even more beds per capita, having 134 and 115 beds per 10,000 people, respectively. This is still far higher than the capacity in developing countries, which is a paltry 12 beds per 10,000 people on average in the bottom quartile of the income distribution. In Appendix Table B.2, we report the availability of intensive care unit (ICU) beds and per capita healthcare costs across a limited set of countries. Consistent with the patterns observed from the number of hospital beds, it appears that low income countries possess significantly fewer ICU beds than high income countries. 


\subsection{More Person-to-Person Contacts and Intergenerational Cohabitation}

COVID-19 is a close contact transmissible disease, and so patterns of social mixing and population density within a community are important determinants of the infection's trajectory. This poses a particularly unique set of challenges for developing countries where, as we show below, there are roughly 25 percent more close contacts in day-to-day activities than in advanced countries. Equally challenging is the greater prevalence of crowded intergenerational households, which substantially increase contacts between vulnerable groups (e.g. the elderly) and the remaining population. In developing countries, the average household size is 5.5, with over 10 percent of households featuring intergenerational cohabitation; in advanced economies, the average household has about 2.5 members and intergenerational cohabitation is rare. ${ }^{1}$

These differences in contact patterns and household structure appear to be an important channel for understanding the transmission of COVID-19, and how targeted policies may succeed or fail at stymying its spread. For instance, large scale studies in South Korea and China find substantial evidence of intra-household transmission, especially by young children infecting older family members when schools are allowed to stay open (Park et al., 2020; Zhao et al., 2019). We incorporate these considerations into our model through the use of contact matrices which disaggregate the frequency of close proximity contacts between different age groups at different locations.

Figure 5 illustrates the data by exhibiting the age-specific contact matrices for advanced and developing countries at four locations: home, work, school, and other. The underlying data is taken from the cross-country surveys of Prem et al. (2017) in which a contact is defined as either a skin-to-skin physical-contact or a face-to-face conversation with three or more words exchanged. Each panel reports the average number of contacts between individuals of a given age ( $x$-axis) with those elsewhere in the age distribution ( $y$-axis) at a given location. Darker colors represent a greater number of daily contacts.

The empirical contact matrices illustrate two important differences between advanced and developing economies. First, individuals in developing countries have many more close proximity contacts in day-to-day activities. The greatest difference in the frequency of contacts appears to be between children at school and other locations, and the smallest difference is between prime age adults at work. The second difference is that cohabitation patterns are much less assortative and more crowded in developing countries. The pronounced diagonal entries in the advanced economy's home contact matrix highlights this point; most people in advanced

\footnotetext{
${ }^{1}$ Intergenerational cohabitation refers to households that have both members under age 15 and over age 60. See Appendix Figure A.3 for how household size and intergenerational cohabitation vary with economic development.
} 
Figure 5: Contact Patterns at Different Locations, Advanced and Developing Economies

(a) Contact Matrices at Home
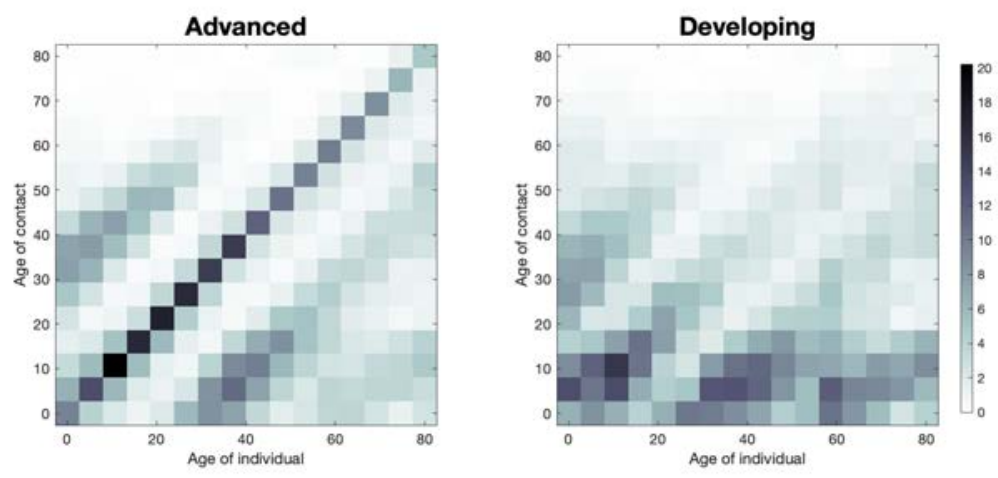

(b) Contact Matrices at Workplace
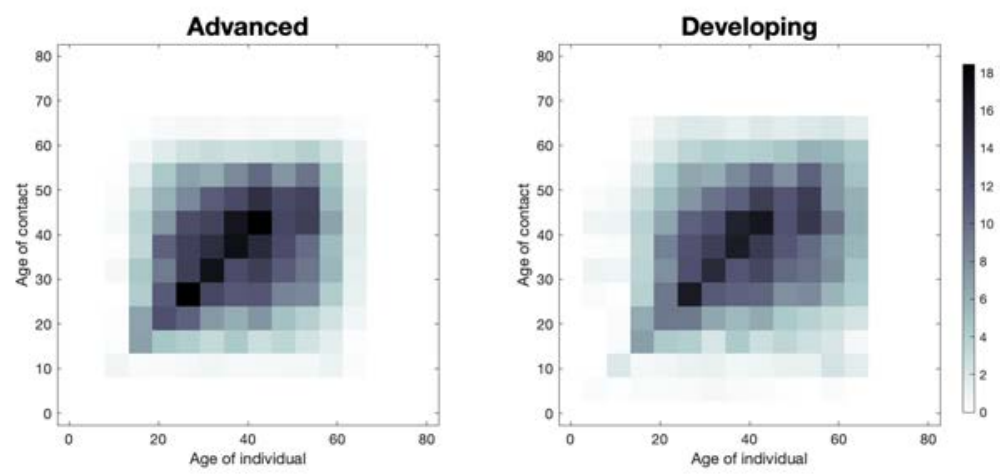

(c) Contact Matrices at School
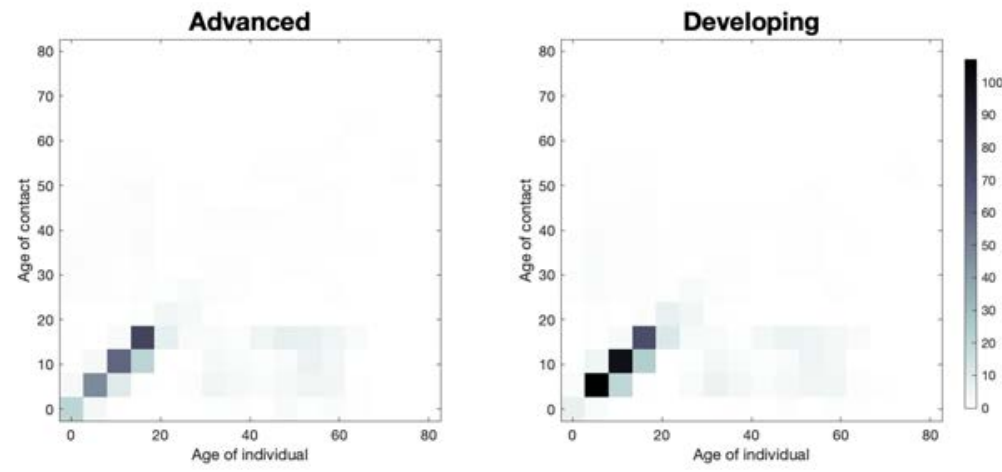

(d) Contact Matrices at Other Locations
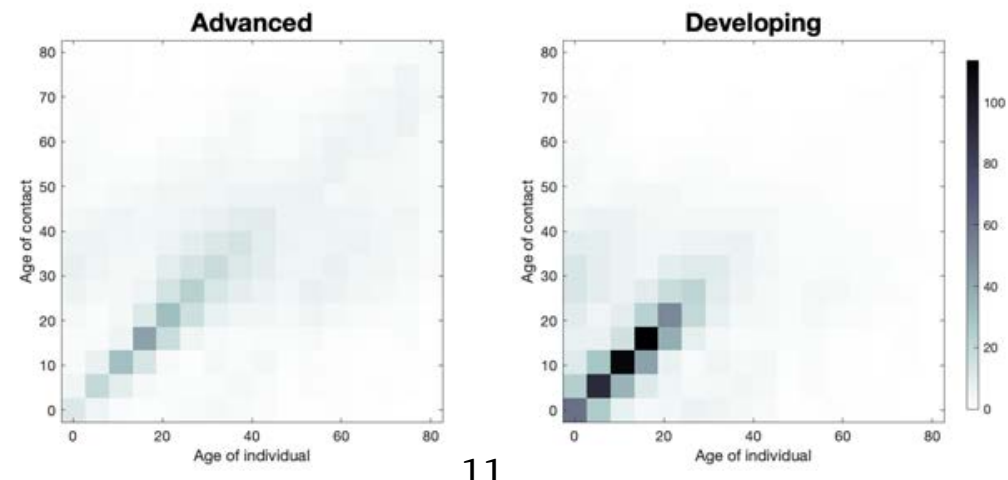
countries live with others in the same age group and the elderly tend to live separately from younger generations, interacting with them only intermittently. Both of these patterns contrast sharply with those in developing countries, which has many more intergenerational interactions within the household, especially between the old and children. Limiting such interactions through social distancing within the home is further complicated by the smaller physical size of dwellings in developing countries. An immediate implication of these cross-country differences in contact patterns is that policies targeting specific locations or sub-populations are likely to have very different impacts in advanced and developing economies, both directly and indirectly.

\section{The Model}

Our analysis draws on a quantitative heterogenous-agent macroeconomic model with epidemiology as in the SICR model to analyze how policy responses to the COVID-19 pandemic should differ in developing countries. The model is equipped with several features that vary between advanced and developing economies that are relevant for the pandemic response, as motivated by the data presented in the previous section. These include uninsurable idiosyncratic health and income risks, age heterogeneity, fiscal capacity constraints, an informal sector, healthcare capacity constraints, and differences in contact patterns. This section now presents these features in detail.

\subsection{Households and Preferences}

The economy is populated by a unit mass of heterogenous individuals who make consumption, savings, and sectoral employment decisions subject to idiosyncratic income and health risks. Individuals differ in their age $j \in\{$ young adult, old adult $\}$, initial assets $a$, and permanent labor productivity $z \sim G$. Time is discrete and each period represents two weeks. Preferences are given by:

$$
U=\mathbb{E}\left[\sum_{t=0}^{\infty} \beta_{j}^{t}\left\{\log \left(c_{t}\right)+\bar{u}\right\}\right],
$$

where the discount factors $\beta_{j}^{t}$ capture age heterogeneity in the population, and $\beta_{\text {young }}<\beta_{\text {old }}$. This specification follows the tractable formulation of Glover et al. (2020) that abstracts from explicitly modeling age, appealing to the logic that pandemics are sufficiently short-lived relative to entire lifetimes. It thus suffices to model only the expected number of years left to live, which is captured by the heterogeneity in discount factors. The term $\bar{u}$ represents the flow utility value of being alive, following the specification of Jones and Klenow (2016), and represents the reason that model households try to avoid fatality risk. Once an individual dies, 
they receive a fixed utility level that potentially depends on their individual characteristics, as we describe below.

Households can choose to work in either the formal $(s=f)$ or informal $(s=i)$ labor markets where they can earn wage $w_{s}$ per effective hour worked. At the beginning of life, workers draw their permanent sectoral productivity, $z \sim G$, and choose occupations as in a Roy (1951) model with one-sided selection. Since work in the informal sector is largely unskilled, we normalize $z$ to unity for this sector so that there is no within sector variation in permanent productivity, as in the specification of Lagakos, Mobarak, and Waugh (2019). We explicitly avoid modeling sectoral differences in working from home, as in Gottlieb et al. (2020), though many of those not able to work from home are likely to be in the informal sector.

Incomes in both sectors are subject to idiosyncratic productivity shocks as in Bewley (1977), Huggett (1993) and Aiyagari (1994). Specifically, we assume that individual labor productivity in each sector is composed of the sector-specific permanent component $z$ and an idiosyncratic component $v$ following the stochastic process:

$$
\log v_{t+1}=\rho_{v} \log v_{t}+\epsilon_{t+1}, \quad \epsilon_{t+1} \sim F\left(0, \sigma_{v}\right)
$$

We include idiosyncratic income risk because developing countries are far from having full insurance, and so accounting for how people insure themselves in response to policies which may keep them away from work for prolonged periods of time is a first order consideration.

After choosing their occupation and observing their income realization, households make consumption and savings decisions given the interest rate, $r$, and subject to a no-borrowing condition, $a \geq 0$. Formally, the household budget constraint is given by:

$$
c+a^{\prime} \leq \mathbb{1}_{\{s=i\}} w_{i} v+(1-\tau) \times \lambda_{L D}^{w} \times \mathbb{1}_{\{s=f\}} w_{f} z v+(1+r) a+T,
$$

where $\tau$ is the income tax rate and $T$ is government transfers. The term $\lambda_{L D}^{w}$ parameterizes productivity lost during the imposition of a government lockdown and is equal to one in normal times and equal to $\lambda_{L D}^{w}=\lambda_{w}<1$ during lockdowns. Importantly, limited state capacity implies that government taxes and commercial restrictions, such as the lockdown, can only be enforced in the formal employment sector. In reality, enforcement capabilities are probably more nuanced, but it is almost certainly much easier in formal places of business than in informal activities. The possibility of moving into the informal sector in response to a lockdown is similar to the movements out of market activities at the start of the pandemic emphasized in Krueger, Uhlig, and Xie (2020), and broadly consistent with the evidence of Zhao, Storesletten, and Zilibotti (2019) that workers respond to economic downturns by moving back into 
agriculture.

\subsection{Aggregate Production Technology}

The economy produces a single final good by combining domestic and foreign capital with labor services supplied by the formal and informal employment sectors. The aggregate production technology is given by:

$$
Y=L^{\alpha} K^{1-\alpha},
$$

where $0<\alpha \leq 1$ is labor's share of value-added. The aggregate capital stock is composed of both domestic and foreign sources, $K=K^{D}+K^{F}$, which can be rented at an exogenously given international rental rate $r^{F}$ (different from $r$, as we explain below) and which depreciates at rate $\delta$.

Aggregate labor depends on the supply of both formal and informal labor services. Since skilled work is largely concentrated in the formal sector, and unskilled work in the informal sector, it is natural to model the two labor inputs as gross-substitutes in the aggregate (as in Ulyssea, 2018). Formally, aggregate labor supply is given by:

$$
L=\left[A L_{f}^{\frac{\sigma-1}{\sigma}}+L_{i}^{\frac{\sigma-1}{\sigma}}\right]^{\frac{\sigma}{\sigma-1}}
$$

where $0<\sigma<\infty$ is the elasticity of substitution between formal and informal labor services and $A$ indexes the relative productivity of formal sector employment. We allow technology $A$ to augment skilled labor, and not unskilled labor, since a large literature finds that cross-country productivity differences are skill-biased, rather than skill neutral (Caselli and Coleman, 2006; Malmberg, 2018). This assumption is important for the sorting of workers by skill in the model, and the prediction that workers with higher skill (permanent productivity) levels sort into the formal sector, with less productive workers selecting into the informal sector.

\subsection{Credit and Capital Markets}

Credit market incompleteness prevents households from borrowing against future earnings. As a result, individuals must maintain non-negative assets in formulating their consumption plans subject to (3), giving rise to hand-to-mouth consumers as well as a precautionary savings motive in response to idiosyncratic health and income risks. The precautionary motive is important for getting aggregate welfare measurements correct since it creates another feedback between the epidemiological and economic dynamics, as individuals withhold some consumption to increase precautionary savings in response to the pandemic's onset. 
Furthermore, financial frictions in capital markets create a spread between the economy's borrowing and savings rates. Specifically, the interest rate paid on domestic savings is such that $r=r^{F}-\chi$ where $\chi>0$ represents a financial wedge leading the returns on savings to be less than the rental rate of capital faced by governments and firms borrowing in international capital markets. These frictions increase the number of economically vulnerable hand-to-mouth consumers in developing countries relative to advanced ones, raise the cost of government borrowing to support welfare programs, and distort capital accumulation.

\subsection{Public Health and Hospital Capacity}

Households face idiosyncratic health risk which can reduce their labor productivity and increase the probability of dying. Susceptibility to infection is determined in part by economic decisions taken by households. Once infected, progression of the disease depends on an individual's age and the availability of public health infrastructure offering treatments.

Health risks are modeled using an SICR epidemiological model with five health states: susceptible $(\mathbb{S})$, infected $(\mathbb{I})$, critical $(\mathbb{C})$, recovered $(\mathbb{R})$, and deceased $(\mathbb{D})$. We denote by $N_{t}^{x}$ the mass of individuals in each health state $x \in\{\mathbb{S}, \mathbb{I}, \mathbb{C}, \mathbb{R}, \mathbb{D}\}$ at time $t$ and use $N_{t}=N_{t}^{\mathbb{S}}+N_{t}^{\mathbb{I}}+N_{t}^{\mathbb{C}}+N_{t}^{\mathbb{R}}$ to measure the non-deceased population. Figure 6 illustrates how these states evolve:

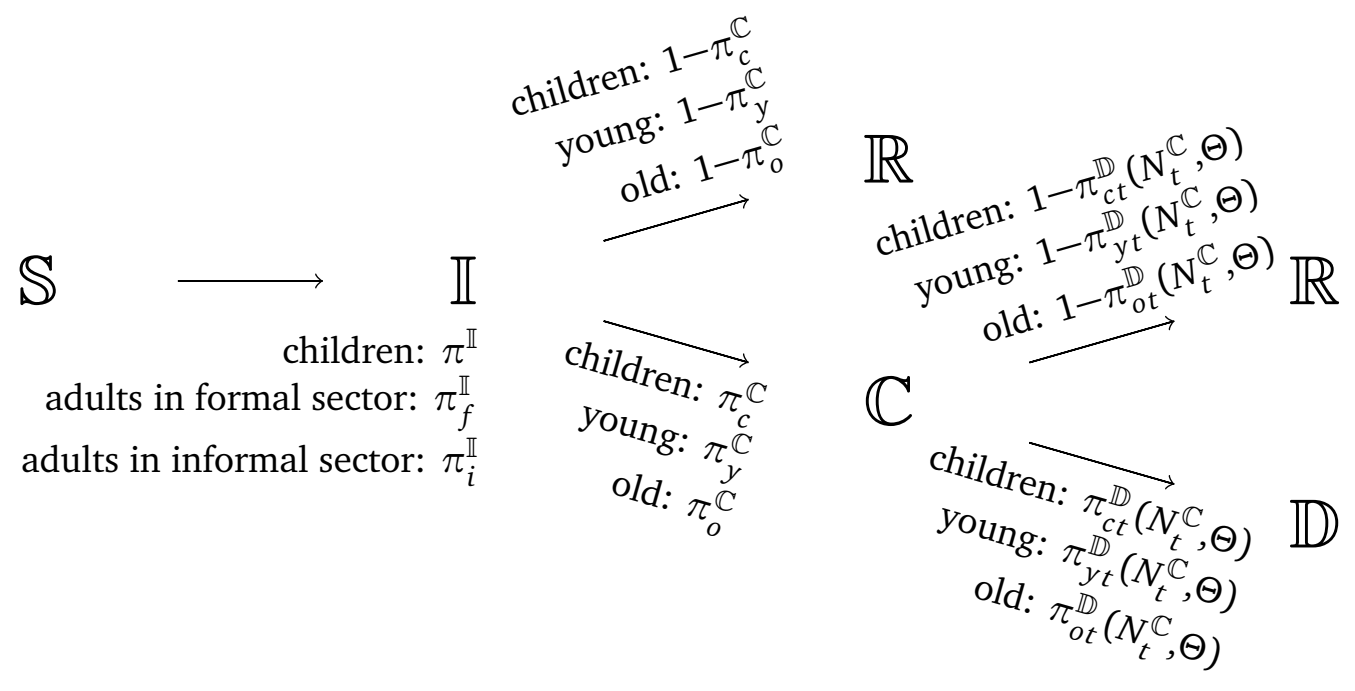

Figure 6: Dynamics of Health States and Transition Probabilities

Individuals who contract the virus experience a proportional drop in productivity of $1-\eta$ for one model period (two weeks), at which point they either recover or enter a critical health state. The probability of becoming critically ill depends on an individual's age and is given by $\pi_{j}^{C}$. Those in critical health are unable to work and require hospitalization. The likelihood of recovery in the hospital depends again on their age in addition to the availability of public 
health infrastructure, such as ICU beds and ventilators. In particular, the fatality rate of a critically ill patient of age $j$ is given by:

$$
\pi_{j t}^{\mathbb{D}}\left(N_{t}^{\mathbb{C}}, \Theta\right)= \begin{cases}\pi_{j}^{\mathbb{D}} & \text { if assigned ICU bed } \\ \kappa \times \pi_{j}^{\mathbb{D}} & \text { if not assigned }\end{cases}
$$

where $\pi_{j}^{D}$ is a baseline fatality rate for age $j$ individuals in critical health and $\kappa$ governs the impact on fatality rates of strained hospital resources. Whether or not a critically ill patient receives an ICU bed depends on overall hospital capacity and the number of other patients. Specifically, letting $\Theta$ denote hospital ICU capacity, the probability a new patient receives an ICU bed is given by $\min \left\{\Theta / N_{t}^{C}, 1\right\}$. In other words, all critically-ill patients receive an ICU bed if hospital capacity constraints are not binding, and beds are rationed amongst the critically-ill with probability $\Theta / N_{t}^{C}$ when constraints bind.

\subsection{Location-Specific Contact Matrices and Virus Contagion}

We model the transmission of COVID-19 using location- and activity-based contact matrices. Disaggregating aggregate mixing patterns in this manner allows us to distinguish how contacts between different segments of the population vary by location, and how each type of contact interacts with various lockdown policies. For example, commercial lockdowns can mitigate the spread of disease at work while school closures reduce contacts between children from different households. Such nuance is necessary for understanding the direct and indirect consequences of any lockdown which does not apply to all individuals in all locations.

We also use the contact matrices to capture the presence of children within households, and how their behavior contributes to the spread of disease. Instead of specifying a child's decision problem, we assume that children attend school full-time (when it's open) and implicitly enjoy consumption and leisure by interacting with family members as well as other children and adults in society at large. The exact nature of these interactions, and how they vary with economic development, is taken from the data and encoded into the child's entries in the contact matrix. This approach provides a parsimonious model of children which can quantitatively account for their contribution to the model's epidemiology under different lockdown policies without the need to add additional state variables.

The use of contact matrices to model social patterns is common in the epidemiology literature, with the first contact matrices estimated by Mossong et al. (2008). Our work draws on disaggregated contact matrices for 152 countries estimated in Prem et al. (2017), which we further group by income level into advanced, middle-income, and developing countries. The 
matrices summarize contact patterns at four primary locations: home, school, workplace, and other. The aggregate contact matrix, $C$, encompasses the contacts at all four locations:

$$
C=\underbrace{\left[\begin{array}{ccc}
c_{c c}^{H} & c_{c y}^{H} & c_{c o}^{H} \\
c_{y c}^{H} & c_{y y}^{H} & c_{y o}^{H} \\
c_{o c}^{H} & c_{o y}^{H} & c_{o o}^{H}
\end{array}\right]}_{C^{H}: \text { Contacts at home }}+\underbrace{\left[\begin{array}{ccc}
c_{c c}^{W} & c_{c y}^{W} & c_{c o}^{W} \\
c_{y c}^{W} & c_{y y}^{W} & c_{y o}^{W} \\
c_{o c}^{W} & c_{o y}^{W} & c_{o o}^{W}
\end{array}\right]}_{C^{W}: \text { Contacts at workplace }}+\underbrace{\left[\begin{array}{ccc}
c_{c c}^{S} & c_{c y}^{S} & c_{c o}^{S} \\
c_{y c}^{S} & c_{y y}^{S} & c_{y o}^{S} \\
c_{o c}^{S} & c_{o y}^{S} & c_{o o}^{S}
\end{array}\right]}_{C^{S}: \text { Contacts at school }}+\underbrace{\left[\begin{array}{ccc}
c_{c c}^{O} & c_{c y}^{O} & c_{c o}^{O} \\
c_{y c}^{O} & c_{y y}^{O} & c_{y o}^{O} \\
c_{o c}^{O} & c_{o y}^{O} & c_{o o}^{O}
\end{array}\right]}_{C^{O}: \text { Contacts at other locations }}
$$

An element $c_{i j}^{L}$ of the contact matrix represents the average number of unique contacts an individual in age group $i$ makes with an individual in age group $j$ at location $L$. Consequently, we may express the probability a susceptible person becomes infected in any location as a function of the number of location-specific contacts with age groups $j$ and their age-specific infection rate. Formally, the probability a susceptible person becomes infected is given by:

$$
\left[\begin{array}{l}
\pi_{c}^{\mathbb{I}} \\
\pi_{y}^{\mathbb{I}} \\
\pi_{o}^{\mathbb{I}}
\end{array}\right]=\left[\begin{array}{ccc}
\beta_{c}^{\mathbb{I}} & 0 & 0 \\
0 & \beta_{y}^{\mathbb{I}} & 0 \\
0 & 0 & \beta_{o}^{\mathbb{I}}
\end{array}\right] \times C \times\left[\begin{array}{c}
N_{c}^{\mathbb{I}} / N_{c} \\
N_{y}^{\mathbb{I}} / N_{y} \\
N_{o}^{\mathbb{I}} / N_{o}
\end{array}\right]
$$

where $N_{j}^{\mathbb{I}} / N_{j}$ is the share of infected people in age group $j$ and $\beta_{j}^{\mathbb{I}}$ is the age-specific behaviorally adjusted transmission rate, which reflects both the disease's biological transmission rate as well as the group's behavioral responses to avoid being infected (i.e. improved hygiene or mask-wearing). Using behaviorally adjusted rates is quantitatively important since public behavioral responses can substantially reduce transmission rates in practice.

While the disease's progression is exogenous, the probability a susceptible person becomes infected depends on endogenous economic decisions and the prevalence of infections in the population. Infection rates can be further mitigated by containment policies, such as lockdowns and school closures. Each containment policy corresponds to a specific restriction on the mixing patterns in the contact matrices above. For instance, school closures imply contacts at schools are eliminated, so that $C^{S}=0$. Similarly, economic lockdowns which shut down commercial activity and reduce the number of contacts at work are captured through restrictions on the workplace $\left(C^{W}\right)$ and other location $\left(C^{O}\right)$ matrices. ${ }^{2}$ Importantly, such shutdowns only affect the formal sector so that population susceptibility to infection depends in part on individual

\footnotetext{
${ }^{2}$ Appendix C contains more details about how each containment policy is modeled via restrictions on the disaggregated contact matrices.
} 
occupational choices. Hence, while individuals working in the informal sector always face the baseline contact matrices, those in the formal sector are exposed to reduced contacts at work during economic lockdowns:

$$
C^{W}= \begin{cases}\lambda_{w} \times C^{W} & \text { if working in the formal sector, }(0 \leq \lambda \leq 1) \\ C^{W} & \text { if working in the informal sector }\end{cases}
$$

Finally, it is important to note that our epidemiological model identifies several aggregate health externalities which contribute to the spread of disease. For example, infection probabilities depend in part on the aggregate population of infected individuals, by age-group, and the types of activities they engage in. Such transmission externalities are compounded by hospital congestion effects which cause the fatality rate to increase as the number of critically ill patients being treated expands. These externalities, and their interaction with economic decisions, creates a margin upon which public health policy, such as lockdowns, could act to improve welfare.

\subsection{Government and Taxation}

The government has power to tax, transfer, and impose economic lockdowns subject to the constraints imposed by limited fiscal capacity and labor market informality. We further require that the government run a balanced flow budget which satisfies,

$$
B_{t}+\Pi+\tau \iint \mathbb{1}_{\{s=f\}} y(a, x, v) d Q d G=\Delta \times T
$$

where $\Pi$ represents natural resource revenue or foreign aid, $y(a, x, v)$ is pretax income for individual $(a, x, v) \sim Q, \tau$ is the prevailing tax rate, and $T$ is aggregate transfers to households. Limited fiscal capacity is captured by the iceberg cost $\Delta>1$, which require the government raise $1 / \Delta$ dollars in revenue for every dollar of transfers to households. Modeling limited capacity through the iceberg costs $\Delta$ is a parsimonious way to represent the resources developing countries lose simply trying to collect taxes and the funds that are diverted to self interested parties before being spent on public programs, as emphasized by the literature on fiscal capacity.

In addition to tax revenue, we allow developing countries access to emergency bonds, $B_{t}$, which can be used to finance additional welfare transfers during government imposed lockdowns. The source of these funds is international donors and multinational institutions such as the IMF, World Bank, and World Health Organization. Funds borrowed for emergency trans- 
fers accrue interest at rate $1+r^{F}$ until the pandemic ends, at which they are repaid through annual annuities. Formally, emergency transfers are given by:

$$
B_{t}= \begin{cases}\bar{B} & \text { during the lockdown } \\ -\frac{r^{F}}{1+r^{F}} \times \sum_{t_{l}-t_{s}}^{t_{l}-t_{e}}\left(1+r^{F}\right)^{t} \bar{B} & \text { after pandemic ends } \\ 0 & \text { otherwise }\end{cases}
$$

where $\bar{B}$ is the size of per-period emergency transfers during lockdown, which we take parametrically, and $t_{s}, t_{e}$, and $t_{l}$ index the lockdown's start, the lockdown's end, and the pandemic's end, respectively.

Alongside its fiscal powers, the government can impose an economic lockdown on the formal sector. While in place, lockdowns reduce disease transmission rates by $1-\lambda_{h}$ and reduces productivity in the formal sector by $1-\lambda_{w}$. The pair $0<\lambda_{w}, \lambda_{h}<1$ can therefore index government lockdown policies, with lower values indicating stricter lockdown measures.

\section{Quantitative Analysis}

In this section, we discuss the calibration strategy, validate the model's fit, and present the counterfactual results for the benchmark lockdown scenarios, age-dependent policies, and school closures. After validating the calibration, we simulate the aggregate effect of the COVID19 pandemic in developing and developed countries with and without aggregate policy responses. Specifically, we study the transition dynamics which emerge when an economy in steady state that is not expecting a pandemic is suddenly hit by the onset of COVID-19 and correctly anticipates its epidemiological dynamics. For each scenario, we report the cumulative changes in welfare, GDP, and aggregate fatalities over the pandemic's duration relative to the pre-pandemic steady state. We also report results on heterogeneity with respect to age and income. The final section reports alternative counter-factual outcomes considering specifically the effectiveness age-dependent policies and school closures.

\subsection{Data Sources and Calibration}

For expositional clarity, we divide the calibrated targets into three broad categories corresponding to those governing economic mechanisms, those controlling epidemiological dynamics, and those delineating differences between advanced and developing countries.

Table 1 reports parameters which govern the core economic dynamics of the model. Population 


\begin{tabular}{clcc} 
Var & \multicolumn{1}{c}{ Description } & Value & Source / Target \\
\hline \hline$r^{F}$ & Exogenous interest rate & 0.0006 & Pre-COVID T-Bills rate 1.5\% \\
$\phi$ & Shape-parameter of Frechet distribution $G$ & 2.7 & Lagakos and Waugh (2013) \\
$\rho_{v}$ & Persistence of idiosyncratic income shock & 0.91 & Floden and Linde (2001) \\
$\sigma_{v}$ & St.Dev of idiosyncratic income shock & 0.04 & Floden and Linde (2001) \\
$\alpha$ & Labor share & 0.6 & Gollin (2002) \\
$\beta_{y}$ & Discount factor for the young & 0.9984 & Glover et al. (2020) \\
$\beta_{o}$ & Discount factor for the old & 0.9960 & Glover et al. (2020) \\
\hline \hline
\end{tabular}

Table 1: Calibration of Economic Parameters

demographics are modeled using age dependent discount factors accounting for differences in the remaining years of life for young and old workers. The age specific discount factors are taken from Glover et al. (2020), and the stochastic income processes are taken from Floden and Lindé (2001), who estimate similar income processes in the United States and Sweden. The distribution of permanent productivity $z \sim G$ in the formal sector is modeled by a Fréchet distribution with shape parameter $\phi$, taken from Lagakos and Waugh (2013). While our formulation allows for imperfect substitutability between employment sectors, we take these to be perfect substitutes for now. Finally, labor's share of income comes from Gollin (2002), and the rental rate of capital is set to the two-week return on pre-COVID Treasury Bills.

Table 2 reports parameters controlling the epidemiological transmission of disease and their interactions with public health infrastructure and lockdown policies. We take parameters governing the fatality infection rates from Glynn (2020) and the rates of infected cases becoming critical from Ferguson et al. (2020). The effect of hospital congestion on disease fatality rates, $\kappa$, is taken from Glover et al. (2020). The productivity penalty of becoming infected, $\eta$, is set to match an 80 percent share of asymptomatic infection cases; such a choice is motivated by the observation that those known to be infected cannot work, and so have productivity of zero, while those who are infected but asymptomatic may continue to work unhindered.

The behavior-adjusted transmission rates are jointly chosen to match time series data on US deaths and seroprevalence data from Spain. In particular, we assume that adults and the elderly face the same infection generating rate but kids may be differentially susceptible. This leaves two parameters to calibrate. We choose the infection generating rate of adults and the elderly to match the total number of COVID deaths in the US on April 29 (four weeks or two model periods after period 1 which we set to April 1st) under lockdown. The infection generating rate for children is chosen to match a seroprevalence rate estimated in Pollán et al. (2020) of 3.4 percent among children compared to 5 percent among adults. Consistent with this evidence, 


\begin{tabular}{clcc} 
Var & \multicolumn{1}{c}{ Description } & Value & Source or Target \\
\hline \hline$\eta$ & Effect of infection on productivity & 0.8 & Asymptomatic cases \\
$\kappa$ & Impact of hospital overuse on fatality & 2 & Glover et al. (2020) \\
$\lambda$ & Lockdown intensity & 0.68 & Bick and Blandin (2020) \\
$\pi_{c}^{\mathbb{C}}$ & Rate of children entering $\mathbb{C}$ from $\mathbb{I}$ & $5.0 \%$ & Ferguson et al. (2020) \\
$\pi_{y}^{\mathbb{C}}$ & Rate of young entering $\mathbb{C}$ from $\mathbb{I}$ & $6.7 \%$ & Ferguson et al. (2020) \\
$\pi_{o}^{\mathbb{C}}$ & Rate of old entering $\mathbb{C}$ from $\mathbb{I}$ & $38.0 \%$ & Ferguson et al. (2020) \\
$\pi_{c}^{\mathbb{D}}$ & Rate of children entering $\mathbb{D}$ from $\mathbb{C}$ & $0.1 \%$ & Glynn (2020) \\
$\pi_{y}^{\mathbb{D}}$ & Rate of young entering $\mathbb{D}$ from $\mathbb{C}$ & $2.7 \%$ & Glynn (2020) \\
$\pi_{o}^{\mathbb{D}}$ & Rate of old entering $\mathbb{D}$ from $\mathbb{C}$ & $9.0 \%$ & Glynn (2020) \\
$\beta_{c}^{\mathbb{I}}$ & Behavior-adjusted transmission rate for children & 0.0109 & Pollán et al. (2020) \\
$\beta_{y}^{\mathbb{I}}$ & Behavior-adjusted transmission rate for adults & 0.0151 & Cumulative Deaths \\
$\beta_{d}^{\mathbb{I}}$ & Behavior-adjusted transmission rate for elderly & 0.0151 & Cumulative Deaths \\
\hline \hline
\end{tabular}

Table 2: Calibration of Epidemiological Parameters

the calibrated values suggest that children are less susceptible to the disease than adults.

The final parameter, $\lambda_{w}$, summarizes the effect of lockdown policies on disease transmission and labor productivity. The productivity loss from lockdown policies, $\lambda_{w}$ is calibrated to match the 32 percent decline in hours worked during the U.S. lockdown, as documented in the weekly labor market surveys of Bick and Blandin (2020). For simplicity, and in the absence of better data, we assume the reduction in contacts are exactly proportional to the observed reductions in labor supply.

We choose not to differentially calibrate the severity of lockdown policies across developing and developed countries, instead allowing cross-country differences to emerge endogenously from agents' optimizing behavior. Furthermore, while conclusive evidence is not yet available, existing cross-country data suggests that instituted lockdown policies were broadly similar, at least in the formal sector. ${ }^{3}$

Table 3 summarizes parameters which vary across advanced and developing countries. Further evidence of such variation is provided in Section 2. Sectoral total factor productivities are

\footnotetext{
${ }^{3}$ For instance, Figure A.4 uses cross-country data from Google's Community Mobility Report to document the average change in residential and workplace mobility during government lockdowns by country's level of economic development. In all cases, lockdown policies lead to a substantial increase in time spent at home and decrease in time spent at work, and the magnitude of these changes far outweighs any changes across a country's level of development. Studies of specific developing countries yield similar results. Figures A.5 and A.6 use high frequency labor market surveys in Ghana during the same period to show declines in total hours worked of 20 to 25 percent during lockdowns, broadly similar to numbers documented in the United States.
} 


\begin{tabular}{clccc} 
Var & \multicolumn{1}{c}{ Description } & Advanced & Developing & Source or Target \\
\hline \hline$A$ & Formal sectors TFP & 3.0 & 0.14 & $1 \%$ labor informality in US \\
$\bar{u}$ & Flow value of being alive & $11.4 \bar{c}^{U S}$ & $11.4 \bar{c}^{D E V}$ & Glover et al. (2020) \\
$\chi$ & Spread b/w borrowing and lending & 0 & $0.66 \%$ & Donovan (2019) \\
$\tau$ & Marginal tax rate & 0.25 & 0.15 & Besley and Persson (2013) \\
$\Delta$ & Iceberg cost in tax collection & 1 & 2.22 & Dzansi et al. (2018) \\
$\bar{B}$ & Lockdown emergency transfers & $6.6 \%$ & $1.5 \%$ & IMF \\
$\omega_{c}$ & Share of children in population & $17 \%$ & $40 \%$ & UN Population Division \\
$\omega_{o}$ & Share of old in population & $19 \%$ & $5 \%$ & UN Population Division \\
$\Theta$ & Hospital capacity per capita & 0.00042 & 0.00011 & Glover et al. (2020) / WHO \\
\hline \hline
\end{tabular}

Table 3: Calibration of Parameters Varying Across Advanced and Developing Economies

chosen to match the extent of labor market informality across levels of development. The financial wedge in capital markets for developing countries is taken from Donovan (2018), whose model matches the low savings rates among poor African households. We take the iceberg costs resulting from low fiscal capacity from the study of tax collection efficiency in Ghana by Dzansi et al. (2018). The tax rates for the advanced and developing countries are taken from Besley and Persson (2013). We normalize away the effect of financial wedges and fiscal iceberg costs in the calibration of advanced economies, setting them to zero and one, respectively. Comparative values of age demographics and exogenous government revenue in the form of aid and natural resources are taken from the World Bank. In particular, the fraction of the elderly (those above 65) is 19 percent in advanced economies but only 5 percent in developing economies. The corresponding fraction of children in developing countries is 40 percent, while in advanced economies it is only 17 percent.

The flow value of life, $\bar{u}$, is calibrated using the value of statistical life (VSL) approach. Following Glover et al. (2020), we set the per-period statistical value of life to $\$ 515,000$ for advanced economies, equal to 11.4 times average US consumption. The value for $\bar{u}$ is then computed so that the behavioral response to a marginal increase in the risk of death is consistent with the VSL. Specifically, we get $\bar{u}$ by solving,

$$
\mathrm{VSL}=\left.\frac{d c}{d \rho}\right|_{E(u)=k, \rho=0}=\ln (\bar{c})-\bar{u}
$$

where $\rho$ is the risk of death and $\bar{c}$ is average consumption. Absent better evidence, we assume the VSL has unitary income elasticity and adjust $\bar{u}$ for developing countries accordingly.

For advanced economies, we calibrate the size of emergency transfers, $\bar{B}$, to reflect the fiscal stimulus spending during the lockdown for countries in the top quartile of the world income 
distribution. Data on the size of fiscal measures as a percent of GDP come from the IMF's COVID-19 policy tracker. ${ }^{4}$ These programs totaled about 6.6 percent of annual GDP for advanced economies. We similarly calibrate the developing country transfers to match fiscal spending in the bottom quartile of world GDP. While there is more heterogeneity in the developing world, the announced fiscal stimulus programs of countries in the bottom quartile of the world income distribution is about 1.5 percent of annual GDP, roughly one-fourth the size of those in advanced economies.

The remaining cross-country parameters to be set govern the ICU hospital capacity in developing and developed countries. One challenge is that while many countries report hospital bed capacity, few developing countries distinguish explicitly between general hospital capacity and ICU capacity in the data. To address this, we assume the ratio of hospital beds to ICU beds is constant across countries, and calibrate $\Theta$ by adjusting WHO data on the availability of hospital beds in the top and bottom quartiles of country income levels (as in Figure 4) by the ratio of hospital beds to ICU beds taken from Glover et al. (2020).

Finally, Table B.1 reports the contact matrices across four different locations in advanced and developing economies. We aggregate the age-location specific contact matrices reported in Prem et al. (2017) within each income group. Since the original location-specific contact matrices in Prem et al. (2017) are 16x16 dimension with age group by 5-year bins, we convert it to $3 \times 3$ matrices by pooling all contacts within the three age groups in our model: children (age 0-14), young adults (age 15-59), and old (age 60+). We then take the average of the contact matrices across countries within the income group. Lastly, for locations other than home, we multiply the number of contacts by 14 to convert them to a two-week frequency as in the model. For the home contact matrix $C^{H}$, we take into account the fact that the contacts taking place at home are likely to be repeated on a daily basis. To account for this, we calculate the propensity of cross-age interactions within the home from the underlying contact matrices and then re-normalize the average household size across countries in different income groups. This procedure highlights the differential contact patterns within household as well as the household sizes between advanced and developing economies.

The resulting contact matrices demonstrate the differential social mixing patterns between advanced and developing economies. Most prominent is the greater frequency of contacts between children and the old at home in developing countries, resulting from their larger household size and greater prevalence of intergenerational cohabitation. There are also a large number of child-to-child contacts at school in developing economies, reflecting large class size and higher teacher-pupil ratios. Social mixing patterns at workplaces are also slightly different,

\footnotetext{
${ }^{4}$ See https://www.imf.org/en/Topics/imf-and-covid19/Policy-Responses-to-COVID-19
} 
Figure 7: Model Fit of Cumulative Deaths in the United States

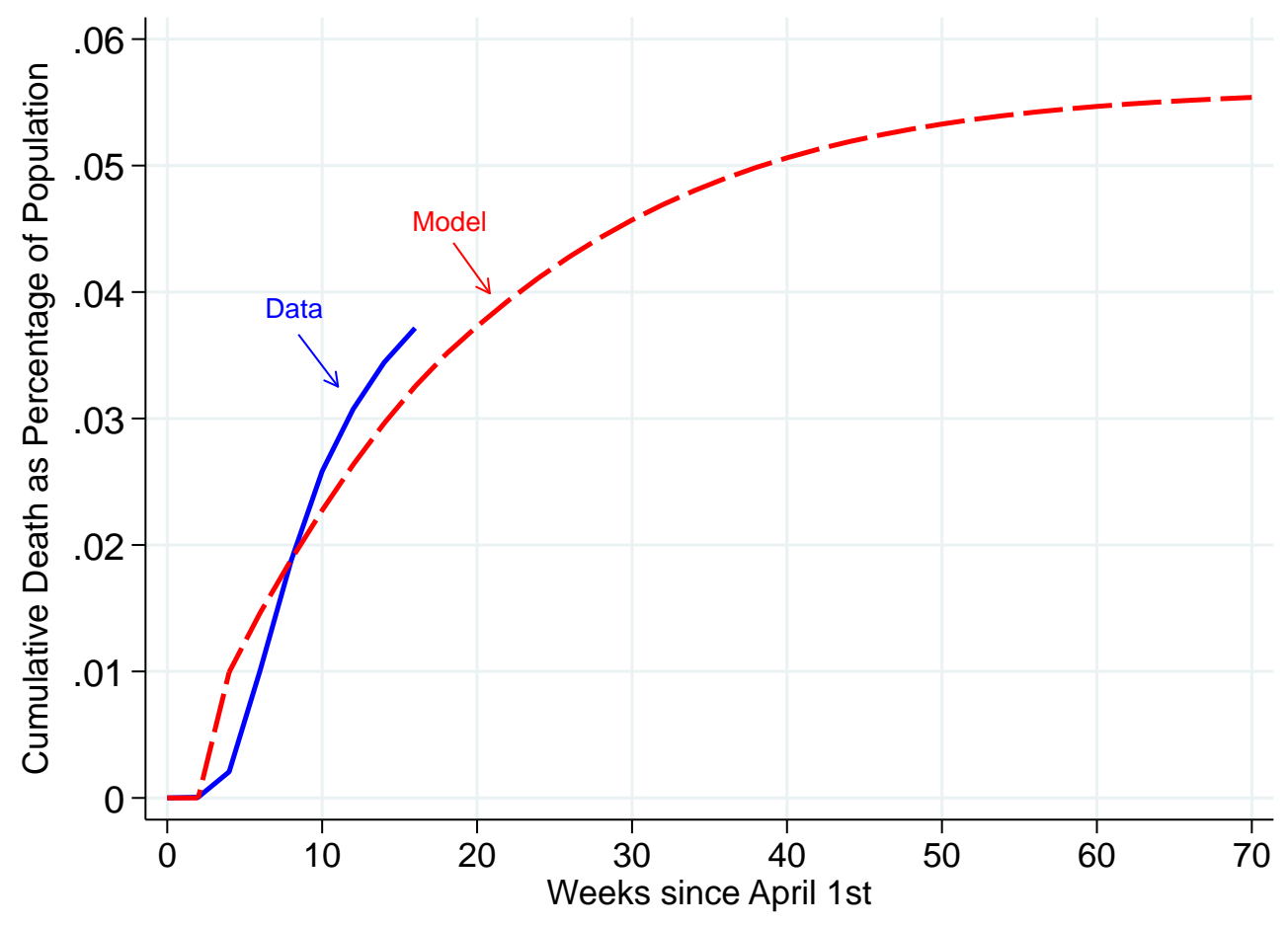

with more contacts between old and young adults in the developing economy, due to higher labor force participation rates of old-age individuals in the developing world.

\subsection{Model Validation}

Before reporting results, we check to ensure that our calibration strategy provides a reasonable fit to relevant moments in the data. We focus specifically on four salient moments crucial to the credibility of subsequent quantitative exercises. These include (1) the relative income levels of advanced and developing countries, (2) the relative size of the informal sector, (3) the fraction of hand-to-mouth consumers, and (4) the epidemiological dynamics of the pandemic.

Overall, in spite of its simplicity, the model does reasonably well at matching key non-targeted moments in low-income countries. It predicts that the fraction of workers employed in the informal sector is 76 percent in the developing economy (rather than 1 percent in the advanced economy) which is consistent with the evidence from Figure 3. The model's predicted fraction of hand-to-mouth households is 19 percent in the advanced economy and 39 percent in the developing economy. Direct analogues from the data are not readily available for the developing economy, though the advanced economy value is in line with the estimates of Kaplan, 
Figure 8: Model Fit of Cumulative Deaths in Brazil

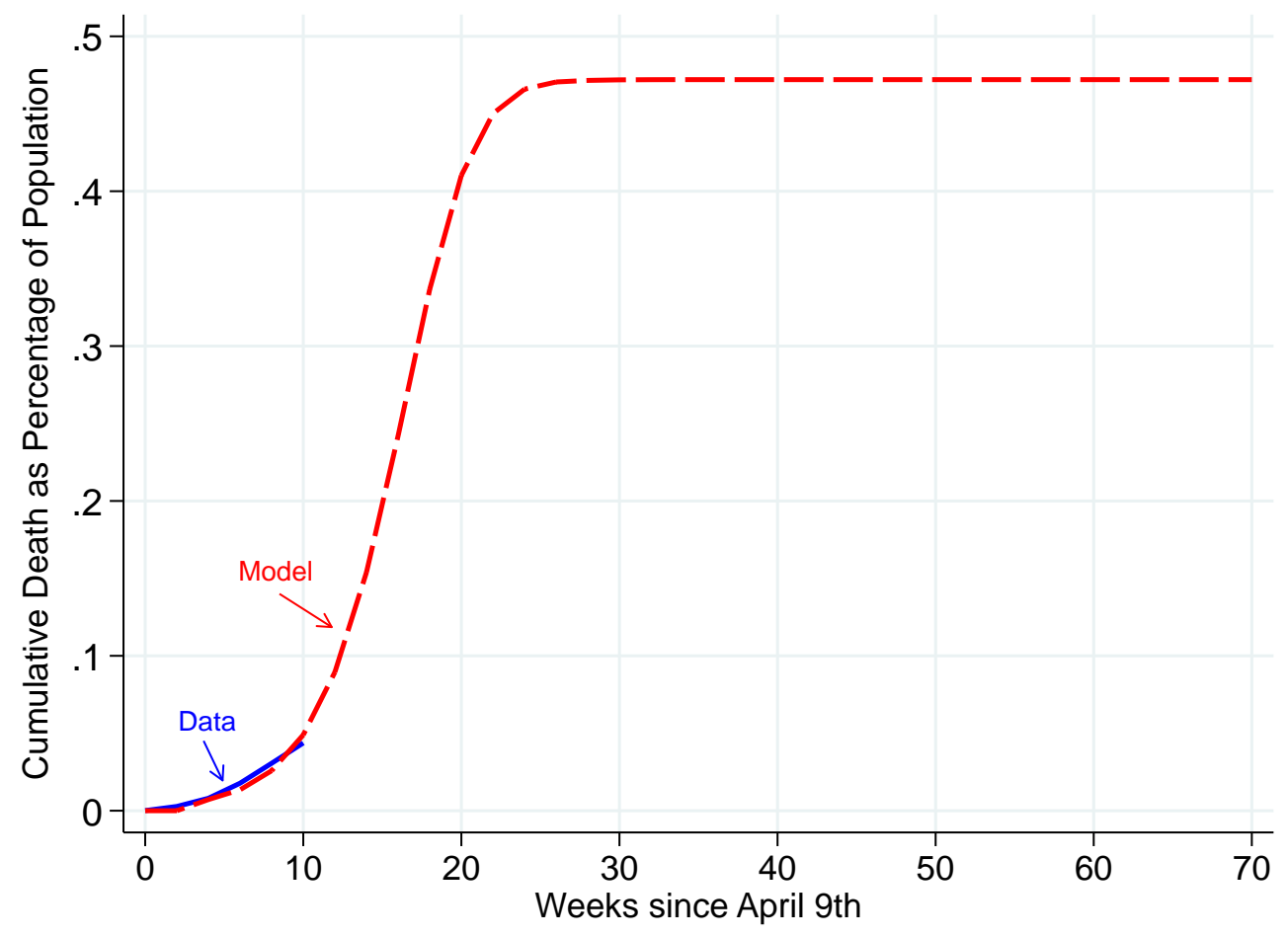

Violante, and Weidner (2014). The level of GDP per capita in the developing economy is 4.4 percent that of advanced economies, or roughly $\$ 2,700$ per year, which is consistent with the average of the bottom quartile of countries.

Figures 7 and 8 provide in- and out-of-sample validation of the epidemiological dynamics of the model by comparing cumulative mortality rates in the model and the data. Figure 7 plots mortality rates in the United States against those predicted by our model. As several of the epidemiological parameters were fit using data from the United States, the figure provides an in-sample validation of our fitting procedure. To ensure the parameters are broadly representative of infection dynamics, we construct additional out-of-sample comparisons with countries that were not directly used in the fitting procedure. A particularly salient example is the case of Brazil which imposed very limited lockdown measures and therefore should exhibit epidemiological dynamics most consistent with the pandemic's underlying dynamics. Figure 8 compares our model's predictions with the data currently available on mortality rates in Brazil. Though the data is more limited, the tight fit of the model and existing data provide further confidence that our calibration accurately captures the most important epidemiological dynamics. 
Table 4: Predicted Effects of the COVID-19 Pandemic

\begin{tabular}{|c|c|c|c|}
\hline & Lifetime Welfare (\%) & GDP (\%) & $\begin{array}{c}\text { Fatalities per 100,000 } \\
\text { People }\end{array}$ \\
\hline \multicolumn{4}{|l|}{ Panel A: Advanced Economies } \\
\hline No Lockdown & -8.9 & -1.3 & 642 \\
\hline Twelve-Week Lockdown & -8.5 & -5.1 & 591 \\
\hline Thirty-Six-Week Lockdown & -7.8 & -12.4 & 477 \\
\hline Seventy-Week Lockdown & -4.4 & -17.6 & 55 \\
\hline \multicolumn{4}{|l|}{ Panel B: Developing Economies } \\
\hline No Lockdown & -3.9 & -1.4 & 339 \\
\hline Twelve-Week Lockdown & -3.8 & -2.5 & 320 \\
\hline Thirty-Six-Week Lockdown & -3.0 & -5.2 & 223 \\
\hline Seventy-Week Lockdown & -2.4 & -7.3 & 164 \\
\hline
\end{tabular}

\subsection{Lockdowns in Advanced and Developing Countries}

Table 4 summarizes results on welfare, GDP, and fatalities under various lockdown policies in advanced, and developing countries. The welfare and GDP entries report the percent change in each outcome variable relative to the country's pre-pandemic steady state levels, and fatalities are reported per hundred thousand people. In our baseline results, we consider aggregate policies that range in length from "no lockdown" to a 70-week lockdown, which lasts through most of the pandemic. We set the maximum duration of the pandemic to 500 days, which is what previous studies have assumed about the amount of time it would take to develop a commercial vaccine. This is, of course, little more than a guess.

The main insight of the counterfactuals is that shorter lockdowns appear to be more effective in developing countries, while longer lockdowns are generally more effective in advanced economies. The result is consistent with reduced-form empirical work which finds that the causal effect of stringent lockdowns is relatively weaker in low income countries (Bharati et al., 
2020). To better grasp the economic intuition, it is useful to first consider what unfolds in each country in the absence of an aggregate policy response. In advanced economies, doing nothing in response to the pandemic results in 642 deaths per hundred thousand, a 1.3 percent contraction in GDP, and a 8.9 percent reduction in consumption-equivalent welfare. ${ }^{5}$ The consequences of doing nothing in lower income countries is markedly less severe. While GDP losses are similar, doing nothing in developing countries leads to 339 death per hundred thousand and a 3.9 percent reduction in welfare - half as much as in advanced economies. The lower costs of inaction in developing countries stems largely from their younger populations whom are intrinsically more resilient to the deleterious effects of the virus, making them less likely to die or lose productivity on account illness.

While developing countries fare better than advanced ones in the absence of an aggregate policy response, they appear less able to effectively mitigate these negative outcomes through lockdown policies. Specifically, while lockdowns always save lives in developing countries, the costs are higher and the benefits more modest. A useful summary statistic to see this is the potency of lockdowns, which we define as the ratio of additional lives saved per hundred thousand over the additional units of GDP lost. Moving from no lockdown to a twelve-week lockdown, advanced economies save an additional 13.4 lives per hundred thousand for each additional unit of lost GDP; in developing economies, short lockdowns save an additional 17.3 lives per hundred thousand for each unit of lost GDP. Under seventy-week lockdowns, for every additional unit of GDP lost saves 36 lives in advanced economies, but only 29.6 in developing ones. These relative effects are largely monotonic across lockdown durations and levels of economic development. In the following section, we unpack the contribution of each economic mechanism in driving these outcomes.

\subsection{Counterfactual Accounting}

To gain a better understanding of the economic forces driving our results, this section analyzes the contribution of each channel in our model to the aggregate consequences of a seventy-week lockdown. While the ultimate duration that lockdowns will remain in effect is currently unknown, our simulations suggest that durations of roughly 70-weeks are necessary to adequately mitigate the spread of disease. To see this more clearly, Figure 9 plots infection trajectories in advanced and developing countries under each of the lockdown durations we consider. In the calibrated model, both twelve-week and thirty-six-week lockdowns provide some mitiga-

\footnotetext{
${ }^{5}$ In reality GDP losses may be even greater than what our model predicts due to features omitted here, such as supply chain disruptions (Bonadio et al., 2020), Keynesian demand channels (Guerrieri et al., 2020), input-output linkages (Baqaee et al., 2020), the labor market disruptions of school closures (Alon et al., 2020), wealth effects through stock market declines (Toda, 2020), or other forces.
} 
Figure 9: Simulated Infection Rates under Different Durations of Blanket Lockdowns

(a) Advanced Economy

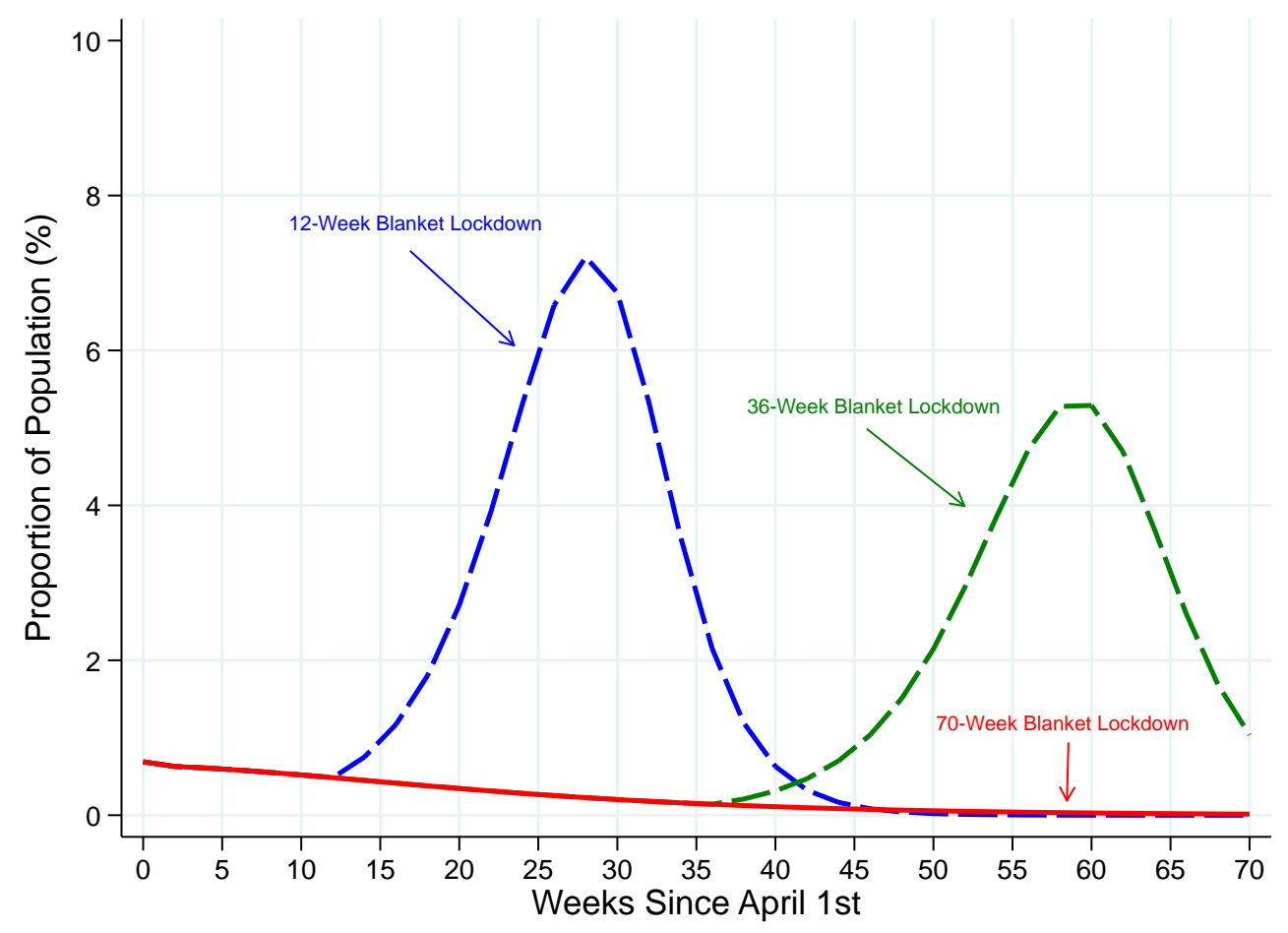

(b) Developing Economy

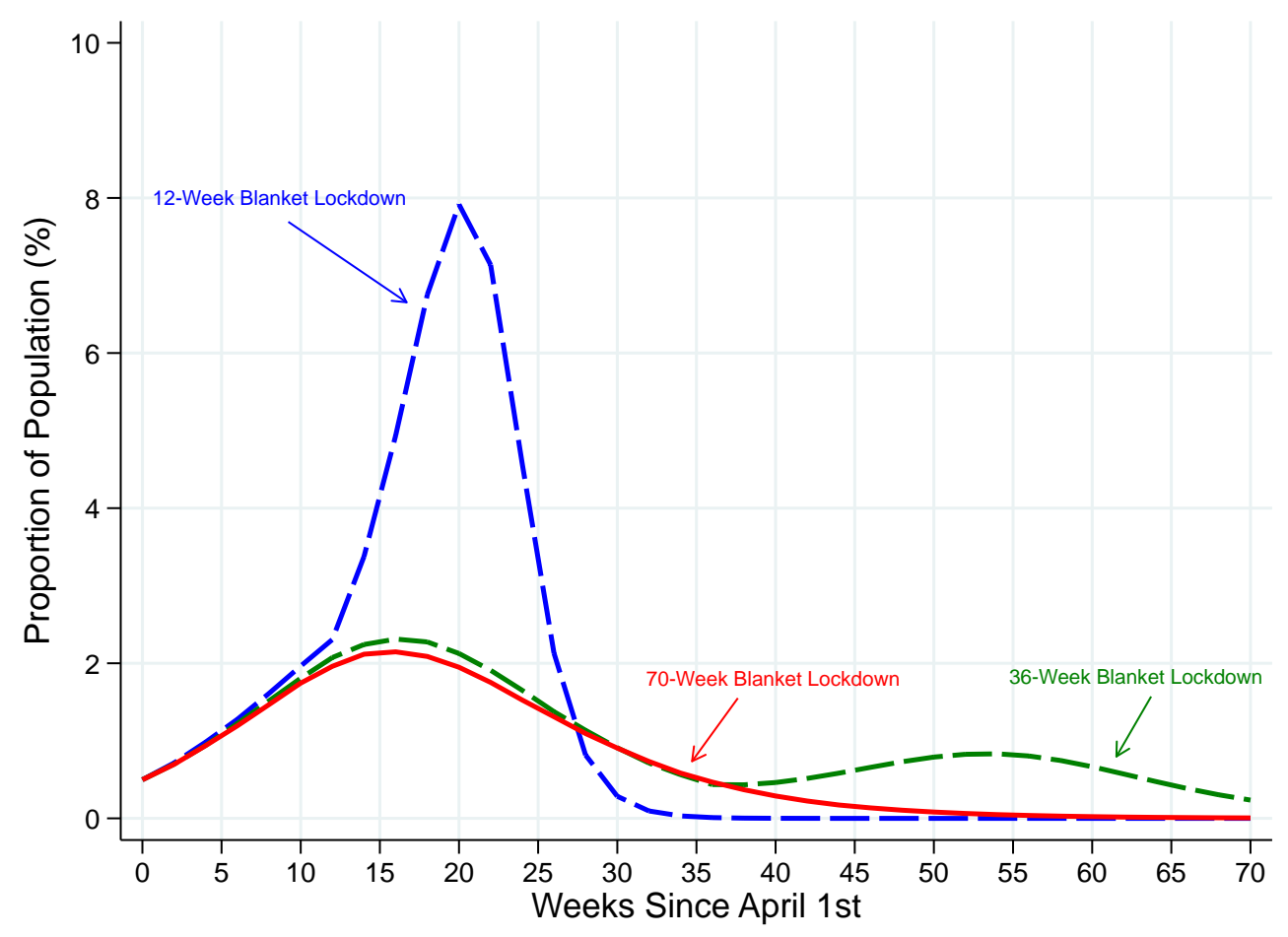


tion while in force, but then lead to a large second-wave of infections after being lifted. The magnitude of the second wave under both policies is smaller in developing countries than in advanced economies. The reason is non-compliance with lockdowns in developing countries. Non-compliance leads to a greater rate of infection while lockdowns are in force, which in turn leads to greater levels of herd immunity early and moderates any second wave of the disease. Under seventy-week lockdowns, both advanced and developing countries are able to slow the spread of the pandemic while also preventing a second wave. Of course in practice, lockdown measures will likely be eased and tightened with economic conditions and the state of public health, making a precise mapping to our model difficult without formally modeling optimal lockdown durations. However, in the absence of such a substantial extension, we consider focusing on seventy-week lockdowns, which adequately slow infections and prevent a second wave, to be a reasonable and parsimonious representation of what will likely unfold in practice.

Given these considerations, Figure 10 reports welfare, GDP, and fatality outcomes under a seventy-week lockdown in the advanced economy as we sequentially endow it with the salient characteristics of developing countries identified in Section 2. The top panel reports counterfactual results for each channel in isolation. Since the magnitude of these individual channels imply substantial equilibrium interactions, the bottom panel adds each mechanism sequentially and cumulates their economic impact.

While all channels play an important role, labor market informality emerges as an especially important channel, both in isolation and through its equilibrium interactions with the other mechanisms. More specifically, the counterfactuals show that differences in fiscal capacity and labor market informality drive most of the differences in output; while contact patterns, age demographics, and informality drive the majority of differences in mortality. The outcomes also highlight the importance of general equilibrium forces in shaping our results, as the impact of each channel in isolation often differs from its effect when considered jointly with the other mechanisms. This observation motivates the necessity of modeling-based approaches to studying the consequences of various COVID-19 policies.

In determining output losses, the distinct importance of informality stems from the fact that it allows for endogenous self-selection into non-compliance with lockdowns. In developing countries, many individuals will find the economic hardships associated with sustained lockdowns to be relatively more damaging than the health risks of not complying. Consequently, while compliance may be relatively good in developing countries at the beginning of a lockdown, over time it erodes as more individuals choose to enter the informal sector in order to circumvent lockdown measures and offset some of the earnings losses it imposes. While such self-selection will serve to moderate the output losses (which are reduced by nearly two-thirds through the 
Figure 10: Counterfactual Economies under 70-Week Lockdown
(a) Individual Contributions

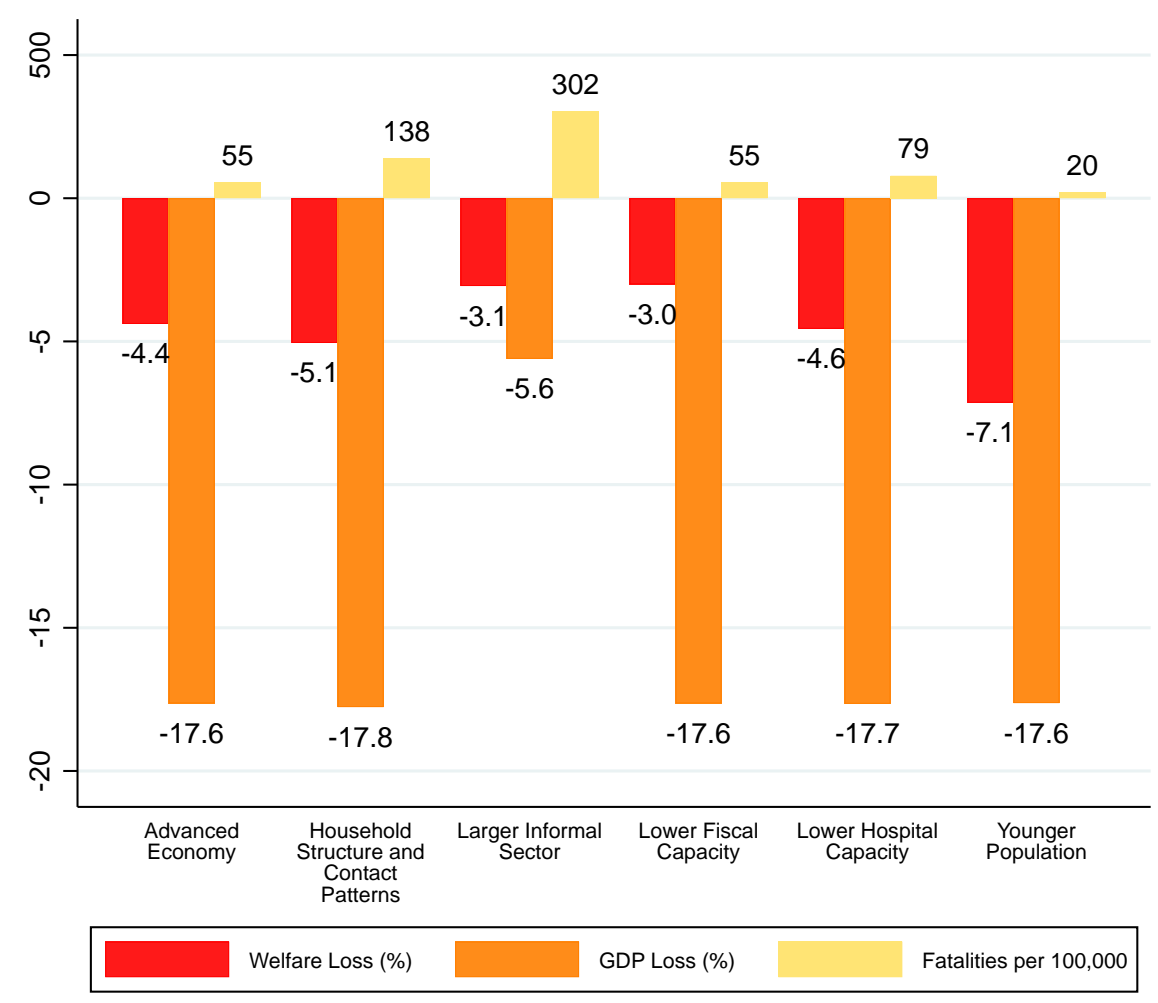

(b) Cumulative Contributions

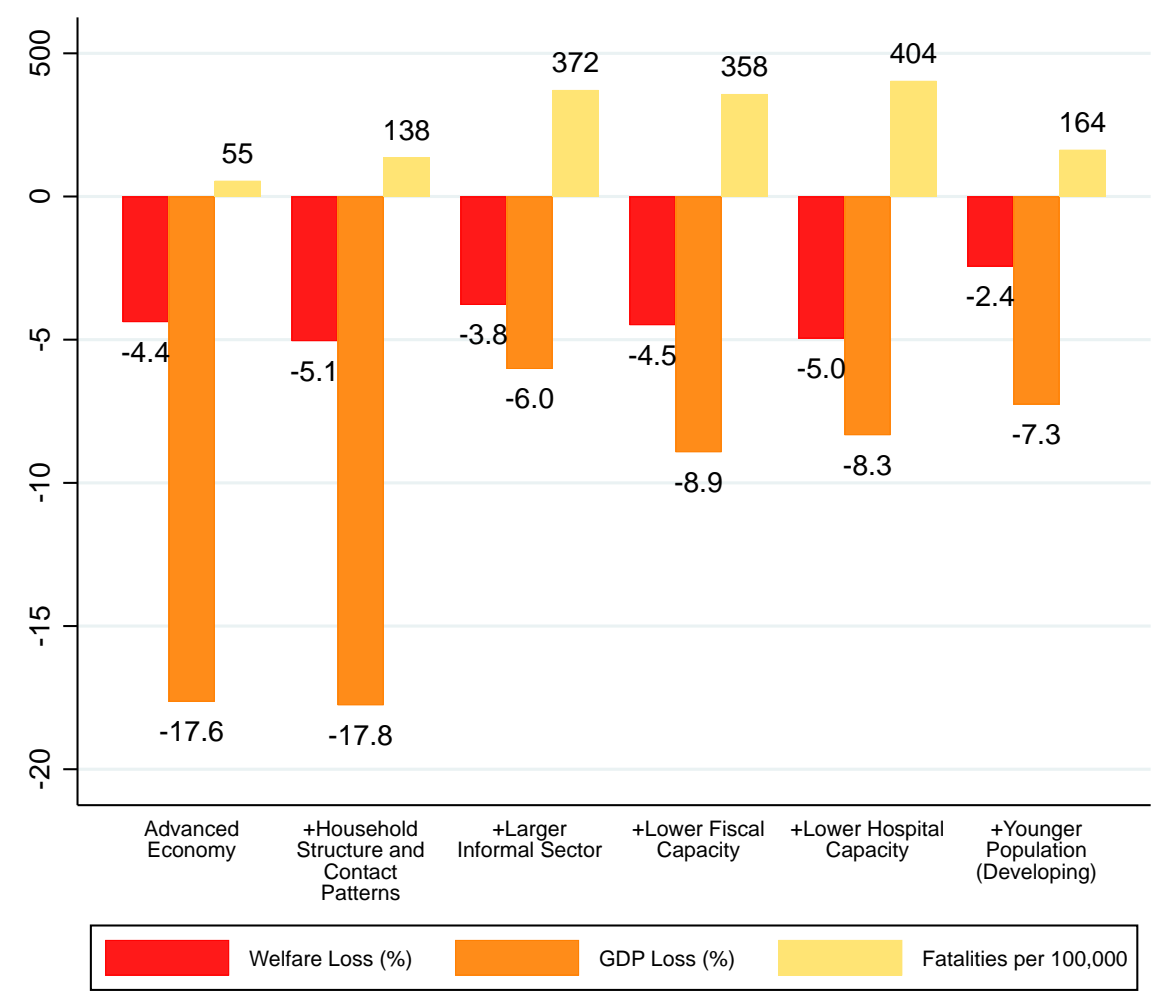


informality channel), it exacerbates public health externalities in the spread of disease, since a large non-complying population will erode the public health benefits to the economy at large. These dynamics are reinforced by weak fiscal capacity in developing countries which prevent large scale transfer programs that could ease economic hardship thereby encouraging compliance. Such non-compliance concerns are less pronounced in advanced economies, where TFP differences between the formal and informal sector are much bigger, households have a greater savings buffer, and fiscal transfer programs are larger and more sustained. An important implication of these results is that developing countries may have to be more conservative than advanced ones when considering the length of their lockdowns.

Turning to mortality rates, differences in demographics and contact patterns appear to play a major role. Endowing advanced countries with the contact patterns and intergenerational cohabitation of developing economies leads mortality rates to nearly triple. The reason is intuitive, as larger households and denser urban centers lead to a greater number of the close contacts in day-to-day activities which spread the disease. It is interesting to note that these patterns of social mixing contribute more to mortality outcomes than do differences in hospital ICU capacity. Despite these negative contributors to mortality, the situation in developing countries is made far less macabre by favorable age demographics. While the first four channels lead the mortality rate in developing countries to be nearly eight times as large as those in advanced economies, accounting for younger populations in developing countries cut prevailing mortality rates by more than half.

In summary, our counterfactual analysis finds meaningful roles for each of the channels in shaping at least one of the outcomes on welfare, GDP, or mortality. Comparing the top and bottom panels of Figure 10 indicates that equilibrium interactions between the various mechanisms in our model are important for understanding their aggregate contributions. This observation motivates the necessity for modeling-based approaches to studying the consequences of various COVID-19 policies. Our current results suggests the most important of these interactions in a developing world context are with the endogenous decisions pertaining to labor market informality, and how those interact with fiscal constraints, demographics, and contact patterns.

\subsection{Age-Dependent Policies and School Closures}

There is already a large and growing body of research documenting the substantial heterogeneity in who bears the costs and benefits of the pandemic and policy responses aimed at tackling it. Such heterogeneity suggests that targeted policies may be more effective than the blanket lockdowns analyzed above. Specifically, while blanket lockdowns impose costs on the entire economy, the benefits accrue overwhelmingly to the old. Age-dependent lockdown poli- 
Table 5: Lives Saved per 100,000 People for every Percentage Point of GDP Lost

\begin{tabular}{|c|c|c|c|c|}
\hline & \multicolumn{2}{|c|}{ Advanced Economy } & \multicolumn{2}{|c|}{ Developing Economy } \\
\hline & $\begin{array}{c}\text { Blanket } \\
\text { Lockdown }\end{array}$ & $\begin{array}{l}\text { Age-dependent } \\
\text { Lockdown }\end{array}$ & $\begin{array}{c}\text { Blanket } \\
\text { Lockdown }\end{array}$ & $\begin{array}{l}\text { Age-dependent } \\
\text { Lockdown }\end{array}$ \\
\hline School Closed & 36 & 74 & 29 & 397 \\
\hline School Open & 19 & 20 & 8 & 22 \\
\hline
\end{tabular}

cies which focus on shielding only the elderly could therefore deliver benefits similar to blanket lockdowns, but at a much lower cost. Such targeted policies have recently been advocated in the public discourse by Ray and Subramanian (2020) and formally analyzed in the context of advanced economies by Acemoglu et al. (2020) and Bairoliya and Imrohoroglu (2020). In principle, such policies may be even more effective in the developing world where the elderly population is smaller and fiscal capacity more limited. On the other hand, the effectiveness of such targeted policies may be undermined by more crowded dwellings and a greater prevalence of intergenerational households, which renders shielding the old from the remaining population infeasible.

To systematically evaluate these countervailing forces, we analyze how aggregate outcomes change in our model when lockdown policies are targeted exclusively at the old. To facilitate comparability, we assume that the lockdown technology parameters are the same, but do not apply to the young population, and keep fixed the total amount of money spent on emergency transfers, $B_{t}$, simply redistributing the excess resources exclusively to the old population.

The first row of table 5 compares the potency of our benchmark blanket lockdown with an age-dependent variant for advanced and developing economies. The results show that age dependent policies greatly increase the efficacy of lockdown programs in both countries when assessed by their potency - the ratio of additional lives saved per hundred thousand over the units of lost GDP. Using this metric, the benefits are especially large in developing countries, whose gains are over six times as big as those in advanced economies. One reason age-dependent policies are so effective in developing countries is that they leverage a unique strength of lowincome countries-their relatively small elderly population-to overcome one of their relative weaknesses-lower fiscal capacity.

The benefits of age-dependent policies, while large, are not unconditional; they depend crit- 
Figure 11: School Closures and Aggregate Infection Dynamics

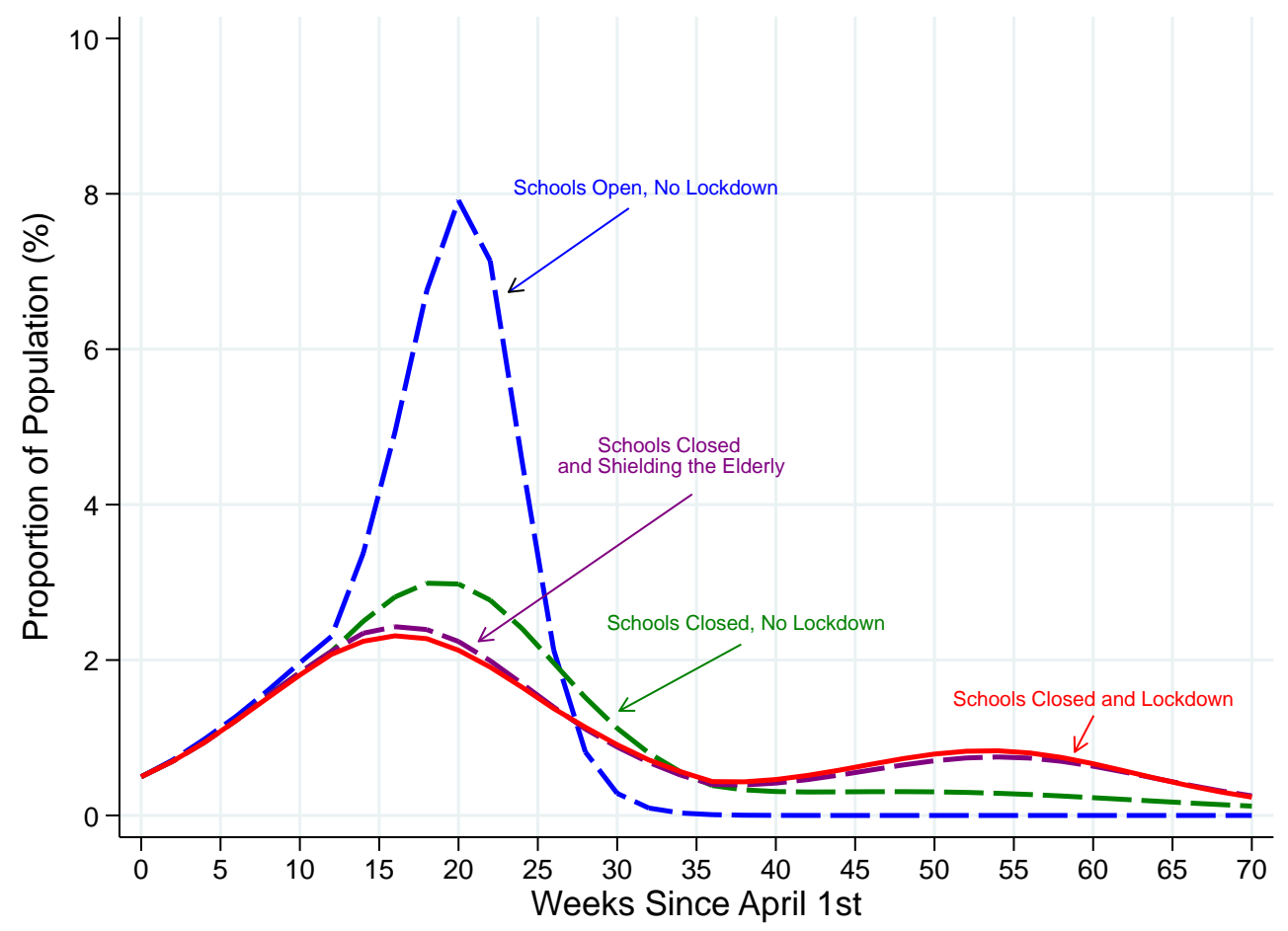

ically on complementary measures which reinforce their ability to shield the old. A specific concern then for developing countries is if and how this can be effectively done in the presence of larger, intergenerational households. Quantitatively, the largest challenge appears to be preventing children, who easily become infected in crowded schools, from infecting their grandparents at home. The empirical contact matrices in Section 2.5 suggest that such concerns are much less pronounced in advanced economies, where schools are less crowded, households are smaller, and cohabitation of grandparents and grandchildren is rare.

To understand the quantitative importance of these considerations, the second row of table 5 considers the impact of lockdown policies if schools remain opened. In both advanced and developing countries, allowing schools to reopen greatly diminishes the benefits of lockdown, especially age-dependent ones. For instance, the model predicts that keeping schools closed during a blanket lockdown saves an additional 17 lives per hundred thousand for each unit of GDP lost in advanced economies, and an additional 21 lives per hundred thousand in developing economies; under age-dependent lockdowns, school closures in advanced economies saves an additional 54 lives, while in developing countries they save an additional 375.

School closures are so effective in developing countries because schools are generally more 
crowded and intergenerational cohabitation is much more common. Together, these characteristics substantially increase the risk that children become infected at school and transmit the virus to their parents or grandparents at home. In fact, these risks are so substantial in developing countries that school closures account for most of the reduction in infections under our benchmark lockdowns.

Figure 11 illustrates the point by plotting the trajectory of aggregate infections in a developing economy, with and without school closures, under 36-week duration policies. The importance of school closures in mitigating the spread of infection is readily apparent, and implies large effects on cumulative mortalities. For instance, our simulation predicts that in a developing country like Nigeria - Africa's most populous - keeping schools closed for 36-week will save roughly 175,000 lives. Furthermore, even though school closures effect predominantly children, nearly all of the additional lives saved are amongst adults and the elderly, consistent with the household linkages we emphasize (see Figure A.7). While the gains of keeping schools closed for 36-weeks appear large, the additional benefits of extending them beyond that are far more modest. The reason is that our model predicts that by this point, many developing countries will be close to achieving herd immunity. This prediction is consistent with a recent large-scale seroprevalence survey by India's Brihanmumbai Municipal Corporation showing that over 40 percent of the population in Mumbai's slums have already been infected (Malani et al., 2020).

From a policy perspective, a main take away is that the benefits of school closures in developing countries may be far greater than in advanced economies. Of course, our current analysis focuses exclusively on the epidemiological benefits of school closures. Not considered are the various costs associated with sustained school closures, such as losses in learning and disruptions to the labor supply of working parents (Alon et al., 2020). Developing countries may even face additional challenges, such as the loss of midday meals for poor families and the viability of students returning to school after large family income shocks stemming from the pandemic. A full accounting of these considerations is beyond the scope of this paper. Nevertheless, our analysis makes clear that the more crowded schools and greater intergenerational cohabitation in developing countries leads to much larger health benefits of school closures. A broader lesson is that accounting for cross-country differences in contact patterns, particularly as they relate to prevailing household structures, is crucial for understanding the aggregate consequences of lockdown policies. 


\section{Conclusion}

This paper provides a quantitive analysis of how lockdown policy should differ between developing and developed economies. Developing economies have distinct characteristics that suggest differing lockdown policies from the west, including younger populations, larger informal sectors, weaker healthcare systems, lower fiscal capacity, more frequent day-to-day contacts between individuals, and a greater prevalence of intergenerational households. Our quantitative macroeconomic model predicts that blanket lockdowns are generally less effective in developing countries, providing more modest welfare gains and generally saving fewer lives per unit of lost economic output. In contrast, age-dependent policies which focus resources on protecting the old are much more potent, as are the public health benefits of school closures which greatly reduce the incidence of intra-household transmission between children and grandparents.

Our quantitative results for welfare, GDP losses, and fatality rates from the pandemic are best viewed as preliminary given that the pandemic is ongoing and there is much that the scientific community still does not know. Our conclusions about the differential effects of COVD-19 between advanced and developing economy may prove more enduring. There can be little doubt that developing economies have vastly younger populations, much larger informal economies, and less fiscal capacity than advanced economies. These basic differences in demographic and economic structure point to optimal lockdown policies that are age-dependent, focusing on keeping just the older population under lockdown, and letting others resume normal economic activity, to the extent that it is possible. Similarly, more crowded schools and a greater prevalence of large intergenerational households in developing countries makes school closures during peak periods of infection an especially effective policy to save lives by reducing the incidence of intra-household transmissions between children and adults. 


\section{References}

Acemoglu, D., V. Chernozhukov, I. Werning, And M. D. Whinston (2020): "A Multi-Risk SIR Model with Optimally Targeted Lockdown," NBER Working Paper No. 27102.

AIYAGARI, S. R. (1994): "Uninsured Idiosyncratic Risk and Aggregate Saving*," The Quarterly Journal of Economics, 109, 659-684.

Aktay, A., S. Bavadekar, G. Cossoul, J. Davis, D. Desfontaines, A. Fabrikant, E. Gabrilovich, K. Gadepalli, B. Gipson, M. Guevara, Et Al. (2020): "Google COVID19 Community Mobility Reports: Anonymization Process Description (version 1.0)," arXiv preprint arXiv:2004.04145.

Alfaro, L., O. Becerra, AND M. Eslava (2020): "EMEs and COVID-19: Shutting Down in a World of Informal and Tiny Firms," NBER Working Paper No. 27360.

Alon, T. M., M. Doepke, J. Olmstead-Rumsey, And M. Tertilt (2020): “The Impact of COVID-19 on Gender Equality," NBER Working Paper No. 26947.

Alvarez, F. E., D. Argente, And F. Lippi (2020): "A Simple Planning Problem for COVID-19 Lockdown," NBER Working Paper No. 26981.

Arellano, C., Y. BAi, AND G. P. Mihalache (2020): "Deadly Debt Crises: COVID-19 in Emerging Markets," NBER Working Paper No. 27275.

Bairoliya, N. AND A. Imrohoroglu (2020): "Macroeconomic Consequences of Stay-at-Home Policies During the COVID-19 Pandemic," COVID Economics Vetted and Real-Time Papers, 13, 71-90.

Baker, S. R., N. Bloom, S. J. Davis, And S. J. Terry (2020): "COVID-Induced Economic Uncertainty," NBER Working Paper No.26983.

BaqAee, D., E. Farhi, M. Mina, And J. Stock (2020): "Policies for a Second Wave," Brookings Papers on Economic Activity, BPEA Conference Drafts, June 25, 2020.

Barnett-Howell, Z. AND A. M. Mobarak (2020): "The Benefits and Costs of Social Distancing in Rich and Poor Countries," Working Paper arXiv:2004.04867.

Berger, D. W., K. F. Herkenhoff, And S. Mongey (2020): “An SEIR Infectious Disease Model with Testing and Conditional Quarantine," NBER Working Paper No. 2690.

Besley, T. And T. Persson (2009): "The Origins of State Capacity: Property Rights, Taxation, and Politics," American Economic Review, 99, 1218-44.

- (2013): "Taxation and Development," in Handbook of Public Economics, Elsevier, vol. 5, 51-110.

BeWley, T. (1977): "The Permanent Income Hypothesis: A Theoretical Formulation," Journal of Economic Theory, 16, 252 - 292. 
Bharati, T., A. M. FAKIR, ET AL. (2020): "Pandemic Catch-22: How effective are mobility restrictions in halting the spread of COVID-19?" COVID Economics Vetted and Real-Time Papers Issue 26, CEPR, 107-136.

Bick, A. AND A. Blandin (2020): "Real Time Labor Market Estimates During the 2020 Coronavirus Outbreak," Unpublished Manuscript, Arizona State University.

Bonadio, B., Z. Huo, A. A. Levchenko, And N. Pandalai-Nayar (2020): "Global Supply Chains in the Pandemic," Unpublished Working Paper, University of Texas at Austin.

Caselli, F. And W. J. Coleman (2006): “The World Technology Frontier,” American Economic Review, 96, 499-522.

ÇAKMaKh, C., S. Demiralp, Ș. Kalemli-Özcan, S. Yesiltas, And M. A. Yildirim (2020): "COVID-19 and Emerging Markets: An Epidemiological Multi-Sector Model for a Small Open Economy with an Application to COVID-19," NBER Working Paper No. 27191.

Chang, R. AND A. Velasco (2020): "Economic Policy Incentives to Preserve Lives and Livelihoods," COVID Economics Vetted and Real-Time Papers, 14, 33-56.

Díez, F., R. Duval, C. Maggi, Y. Ji, I. Shibata, And M. Mendes Tavares (2020): "Options to Support the Incomes of Informal Workers During COVID-19," IMF Research Special Series on COVID-19.

Donovan, K. (2018): "Agricultural Risk, Intermediate Inputs, and Cross-Country Productivity Differences," Unpublished Working Paper, Yale University.

Dzansi, J., A. Jensen, D. Lagakos, I. Otoo, H. Telli, And C. Zindam (2018): "Survey of Local Government Revenue Mobilization Capacity in Ghana, 2017: Summary and Policy Implications," International Growth Centre Project Report S-33417-GHA-2.

Eichenbaum, M. S., S. Rebelo, And M. Trabandt (2020): “The Macroeconomics of Epidemics," NBER Working Paper No. 26882.

Feenstra, R. C., R. InklaAR, And M. P. Timmer (2015): “The Next Generation of the Penn World Table," American Economic Review, 105, 3150-82.

Ferguson, N., D. Laydon, G. Nedjati Gilani, N. Imai, K. Ainslie, M. Baguelin, S. Bhatia, A. Boonyasiri, Z. Cucunuba Perez, G. Cuomo-Dannenburg, ET AL. (2020): "Report 9: Impact of Non-Pharmaceutical Interventions (NPIs) to Reduce COVID-19 Mortality and Healthcare Demand," Unpublished Working Paper, Imperial College London.

Floden, M. AND J. LINDÉ (2001): "Idiosyncratic Risk in the United States and Sweden: Is There a Role for Government Insurance?" Review of Economic Dynamics, 4, 406-437.

Glover, A., J. Heathcote, D. Krueger, And J.-V. RÍos-Rull (2020): “Health Versus Wealth: On the Distributional Effects of Controlling a Pandemic," NBER Working Paper No. 27046.

GlYnN, J. R. (2020): "Protecting Workers Aged 60-69 Years from COVID-19," The Lancet Infectious Diseases; (published online April 16). 
GolLin, D. (2002): “Getting Income Shares Right,” Journal of Political Economy, 110, 458-474.

Gottlieb, C., J. Grobovsek, M. Poschke, And F. Saltiel (2020): "Lockdown Accounting," IZA Discussion Paper No. 13397.

Greenwood, J., P. Kircher, C. SAntos, And M. Tertilt (2019): "An Equilibrium Model of the African HIV/AIDS epidemic," Econometrica, 87, 1081-1113.

Guerrieri, V., G. LORENZONI, L. Straub, AND I. WERning (2020): "Macroeconomic Implications of COVID-19: Can Negative Supply Shocks Cause Demand Shortages?" NBER Working Paper No. 26918.

HuggetT, M. (1993): "The Risk-Free Rate in Heterogeneous-agent Incomplete-Insurance Economies," Journal of Economic Dynamics and Control, 17, 953-969.

ICTD/UNU-WIDER (2019): "Government Revenue Dataset," https://www.wider. unu.edu/project/government-revenue-dataset, Last accessed on 202005-14.

Jensen, A. (2019): "Employment Structure and the Rise of the Modern Tax System," NBER Working Paper No. 25502.

Jones, C. I. AND P. J. KLENOW (2016): "Beyond GDP? Welfare across Countries and Time," American Economic Review, 106, 2426-57.

KAPlan, G., B. Moll, AND G. L. Violante (2020): "The Great Lockdown: Macroeconomic and Distributional Effects of Covid-19," Unpublished Manuscript, University of Chicago.

Kaplan, G., G. L. Violante, AND J. Weidner (2014): “The Wealthy Hand-to-Mouth,” Brookings Papers on Economic Activity, 1, 77-153, spring.

KIM, Y. E. AND N. LOAYZA (2020): "Economic Loss from COVID-19 Fatalities Across Countries: A Value of Statistical Life Approach," Unpublished Manuscript, World Bank.

KRUEger, D., H. Uhlig, AND T. XIE (2020): "Macroeconomic Dynamics and Reallocation in an Epidemic," NBER Working Paper No. 27047.

LAGAKos, D., A. M. MobaraK, AND M. E. WAUGH (2019): “The Welfare Effects of Encouraging Rural-Urban Migration," Unpublished Working Paper, Yale University.

LAGAKOS, D. AND M. E. WAUGH (2013): "Selection, Agriculture, and Cross-Country Productivity Differences," American Economic Review, 103, 948-80.

Malani, A., M. Mohanan, A. ACHARYA, S. ImAD, AND P. Hingorani (2020): "SARS-COV2 Seroprevalence Study in Mumbai: NITI Aayog-BMC-TIFR Study, First Round Report,” Municipal Corporation of Greater Mumbai Public Relations Department Press Release, July 28.

Malmberg, H. (2018): "How Does the Efficiency of Skilled Labor Vary across Rich and Poor Countries? An Analysis Using Trade and Industry Data," Unpublished Manuscript, University of Minnesota. 
Mossong, J., N. Hens, M. Jit, P. Beutels, K. Auranen, R. Mikolajczyk, M. Massari, S. Salmaso, G. S. Tomba, J. Wallinga, et Al. (2008): "Social Contacts and Mixing Patterns Relevant to the Spread of Infectious Diseases," PLoS Med, 5, e74.

Pagés, C., C. Aclan, M. Alfonso, R. Arroio, J. L. Irigoyen, I. Mejía, C. Mendieta, S. Moreno, A. Muente, S. Penaherrera, C. Pombo, F. Regalía, W. D. Savedoff, E. H. STEIN, AND L. TEJERINA (2020): "From Lockdown to Reopening: Strategic Considerations for the Resumption of Activities in Latin America and the Caribbean within the framework of Covid-19," Inter-American Development Bank.

Park, Y. J., Y. J. Choe, O. PARK, S. Y. PArk, Y.-M. Kim, J. Kim, S. KweOn, Y. Woo, J. Gwack, S. S. KIM, ET AL. (2020): "Contact Tracing during Coronavirus Disease Outbreak, South Korea," Emerging Infectious Diseases; (published in online July 16), 26.

Pollán, M., B. Pérez-Gómez, R. Pastor-Barriuso, J. Oteo, M. A. Hernán, M. PérezOlmeda, J. L. SANMARtín, A. Fernández-García, I. CruZ, N. F. DE LARreA, ET AL. (2020): "Prevalence of SARS-CoV-2 in Spain (ENE-COVID): a Nationwide, Population-Based Seroepidemiological Study," The Lancet; (published online July 06).

Prem, K., A. R. CoOK, AND M. Jit (2017): "Projecting Social Contact Matrices in 152 Countries Using Contact Surveys and Demographic Data," PLoS Computational Biology, 13, e1005697.

PRIN, M. AND H. WUNSCH (2012): "International Comparisons of Intensive Care: Informing Outcomes and Improving Standards," Current Opinion in Critical Care, 18, 700.

RAY, D. AND S. Subramanian (2020): “Covid-19: Is There a Reasonable Alternative to a Comprehensive Lockdown?" Ideas for India, March 28.

Roy, A. (1951): "Some Thoughts on the Distribution of Earnings," Oxford Economic Papers, 3, 135-146.

TodA, A. A. (2020): "Susceptible-Infected-Recovered (SIR) Dynamics of COVID-19 and Economic Impact," COVID Economics Vetted and Real-Time Papers Issue 3, CEPR, 43-63.

UlysseA, G. (2018): "Firms, Informality, and Development: Theory and Evidence from Brazil," American Economic Review, 108, 2015-47.

von Carnap, T., I. Almås, T. Bold, S. Ghisolfi, And J. SAndefur (2020): "The Macroeconomics of Pandemics in Developing Countries: an Application to Uganda," COVID Economics Vetted and Real-Time Papers Issue 27, CEPR, 104-122.

Zhao, B., K. Storesletten, And F. Zilibotti (2019): "Business Cycles During Structural Change: Arthur Lewis? Theory from a Neoclassical Perspective," Unpublished Working Paper, Yale University. 


\section{Appendix}

\section{A. Appendix Figures}

Figure A.1: Fraction of the Population Older than Age 65

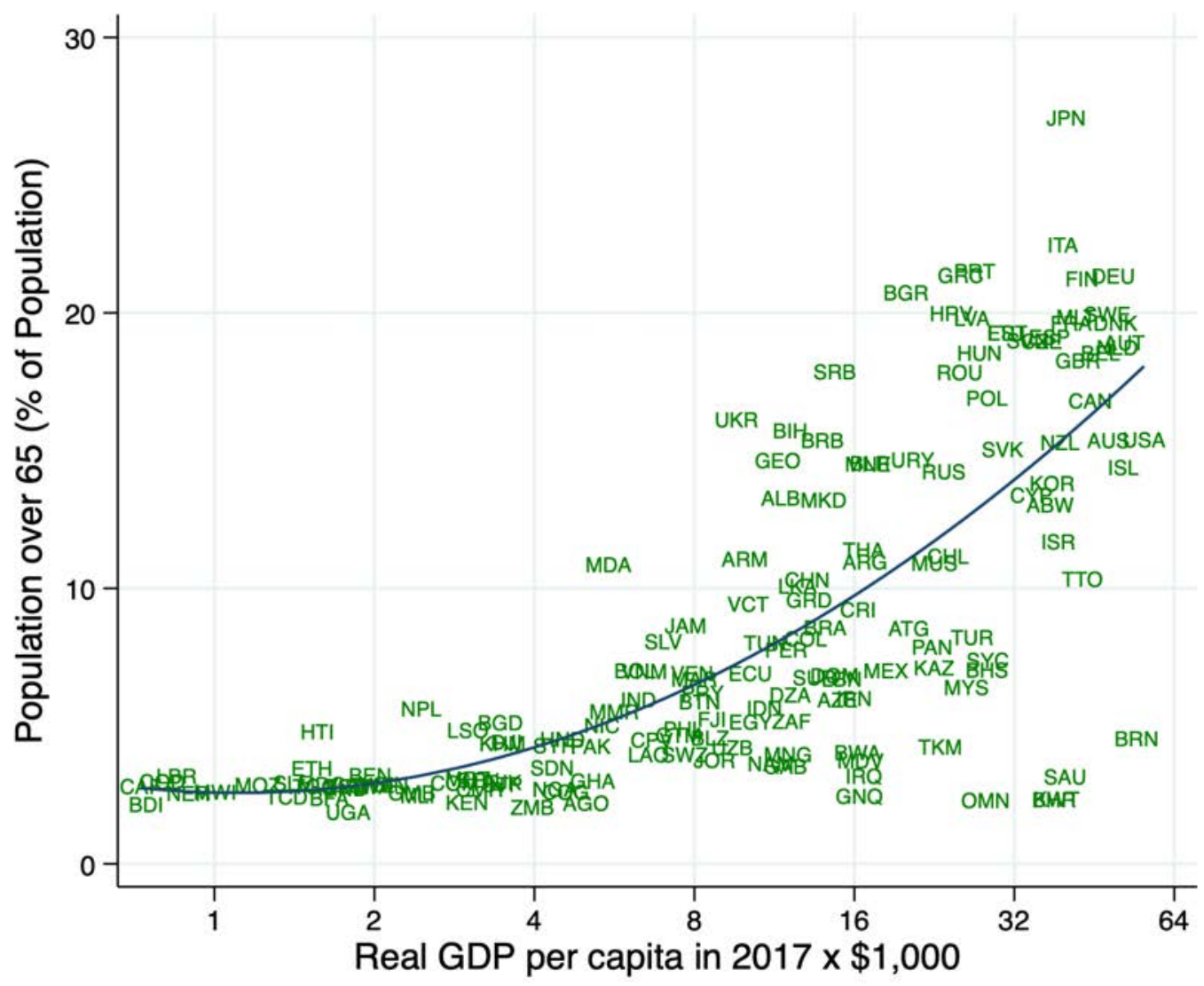

Note: This figure plots the proportion of population ages over 65 and above as a percentage of total population across 162 countries. GDP per capita is from Penn World Table 9.1 (Feenstra et al., 2015). Population data is World Bank staff estimates using the World Bank's total population and age/sex distributions of the United Nations Population Division's World Population Prospects: 2019 Revision. 
Figure A.2: Tax Capacity and Pandemic Spending to GDP

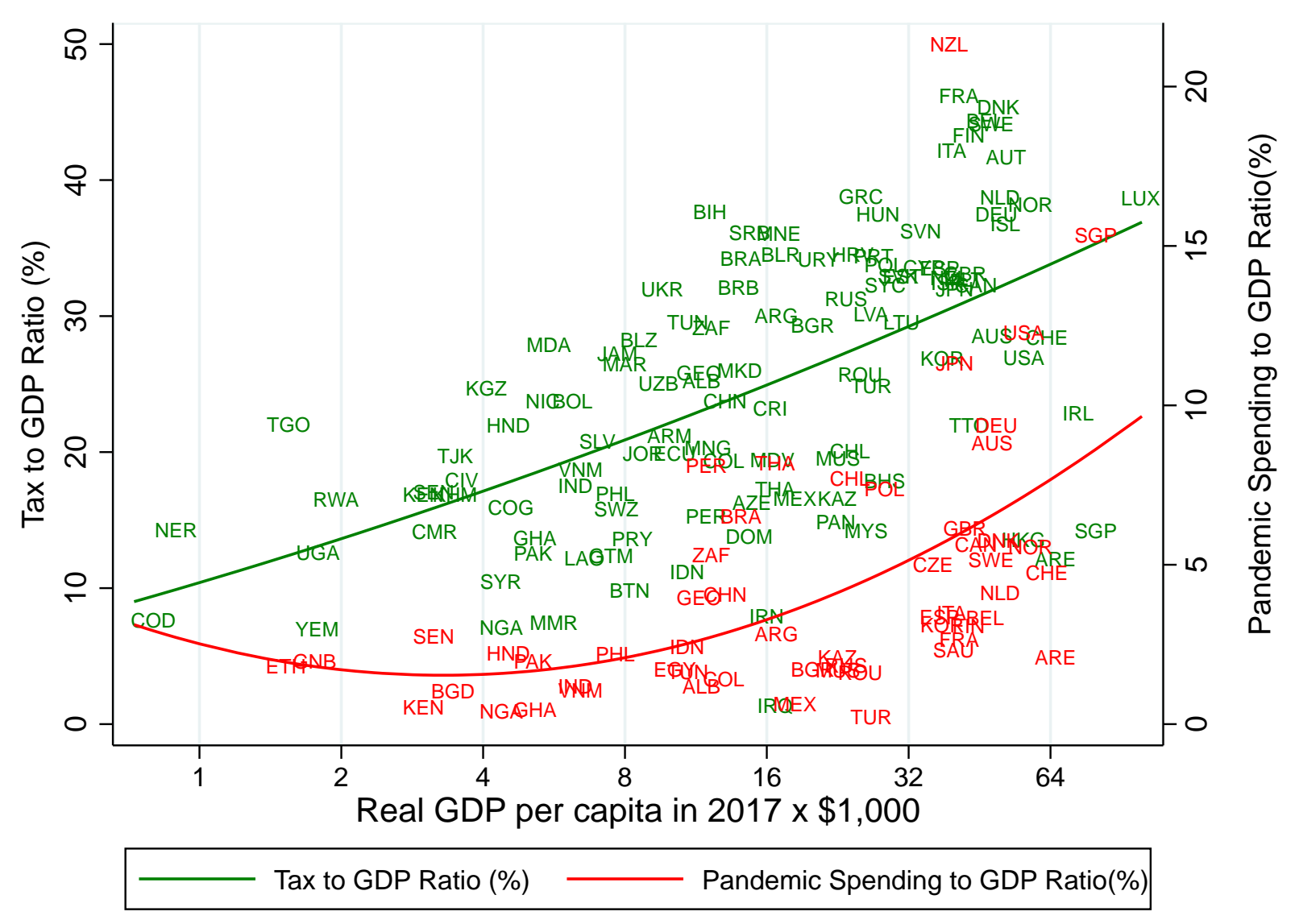

Note: This figure plots the ratio of taxes to GDP and pandemic spending relative to GDP, taken from the IMF. 
Figure A.3: Household Sizes and Intergenerational Cohabitation Patterns

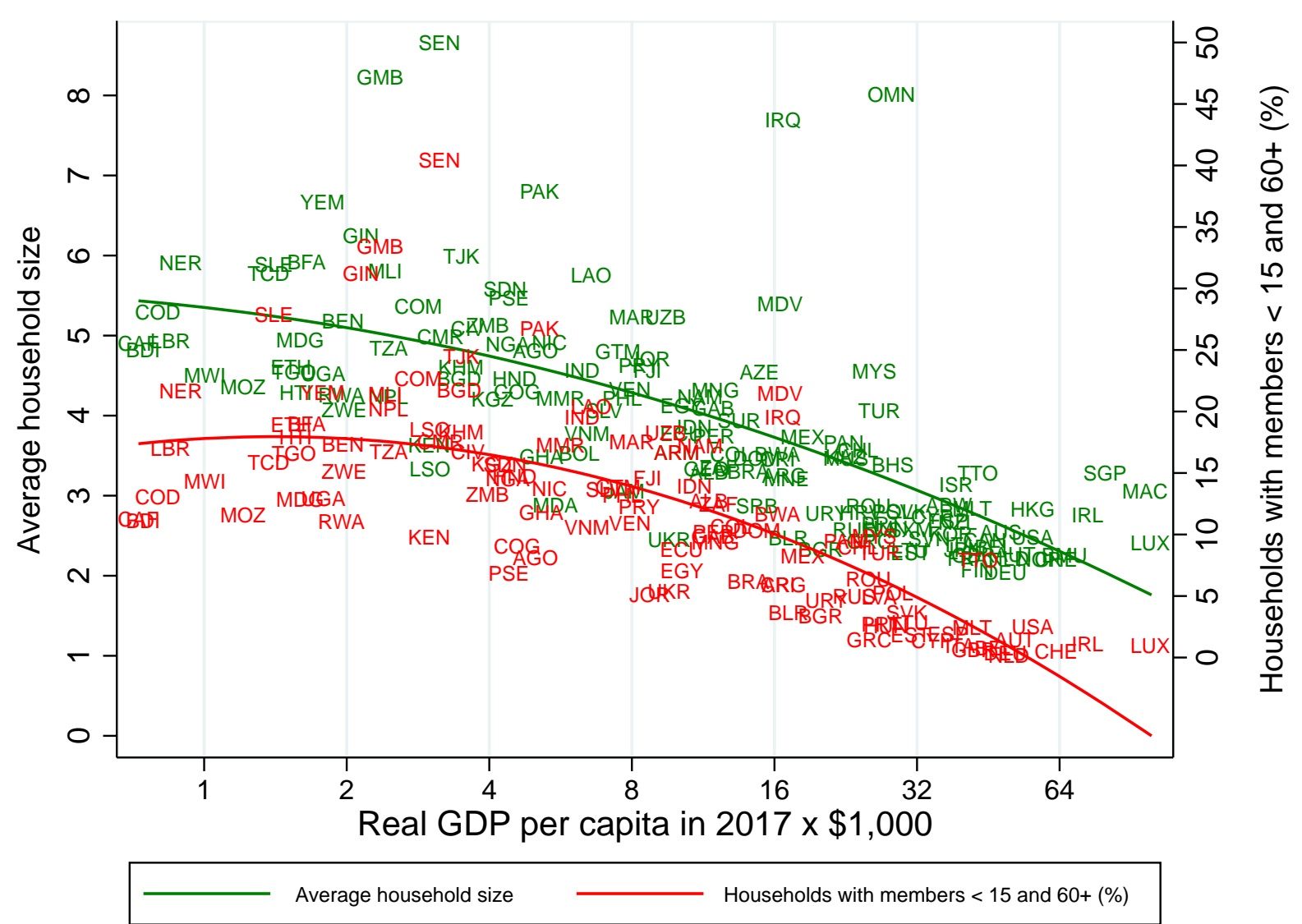

Note: This figure plots the average household sizes and the fraction of households with member(s) under 15 years old and over 60 years old together. 
Figure A.4: Changes in Mobility Across Countries During Lockdown Periods

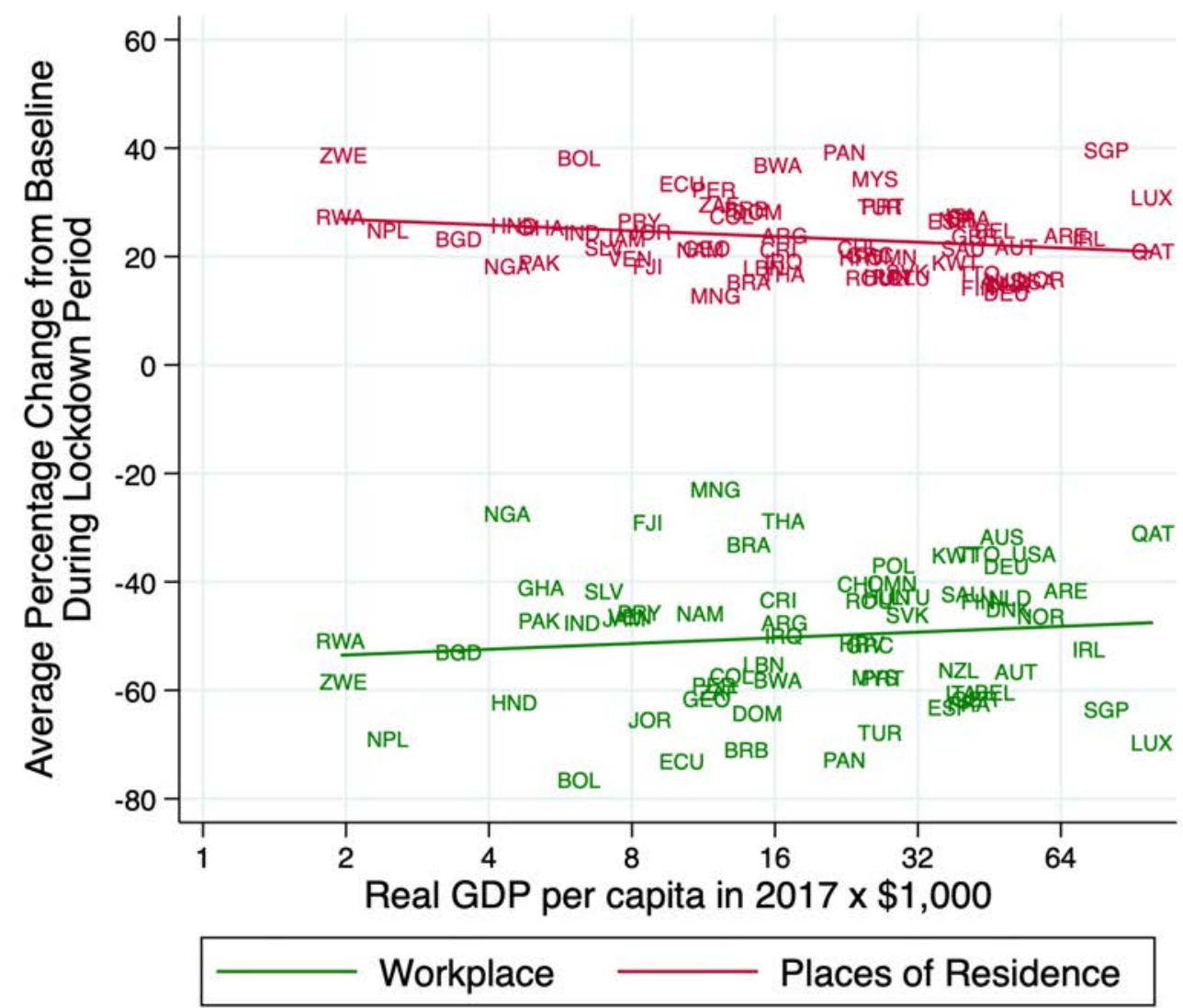

Note: This figure plots the average percentage changes of the mobility metric in the 'Places of Residence' and 'Workplace' categories in the Google Community Mobility Report (Aktay et al., 2020), during the lockdown periods for the 65 countries which had implemented or are implementing lockdown. GDP per capita is from Penn World Table 9.1 (Feenstra et al., 2015). The average across all 65 countries is 23.44 percent. The slope of the fitted line is 1.52 , with $p$-value of 0.354 for the 'Workplace' category. For the 'Places of Residence' category, the slope of the fitted line is -1.52 , with $p$-value of 0.083 . 
Figure A.5: Employment Rate in Ghana Around the Lockdown Period

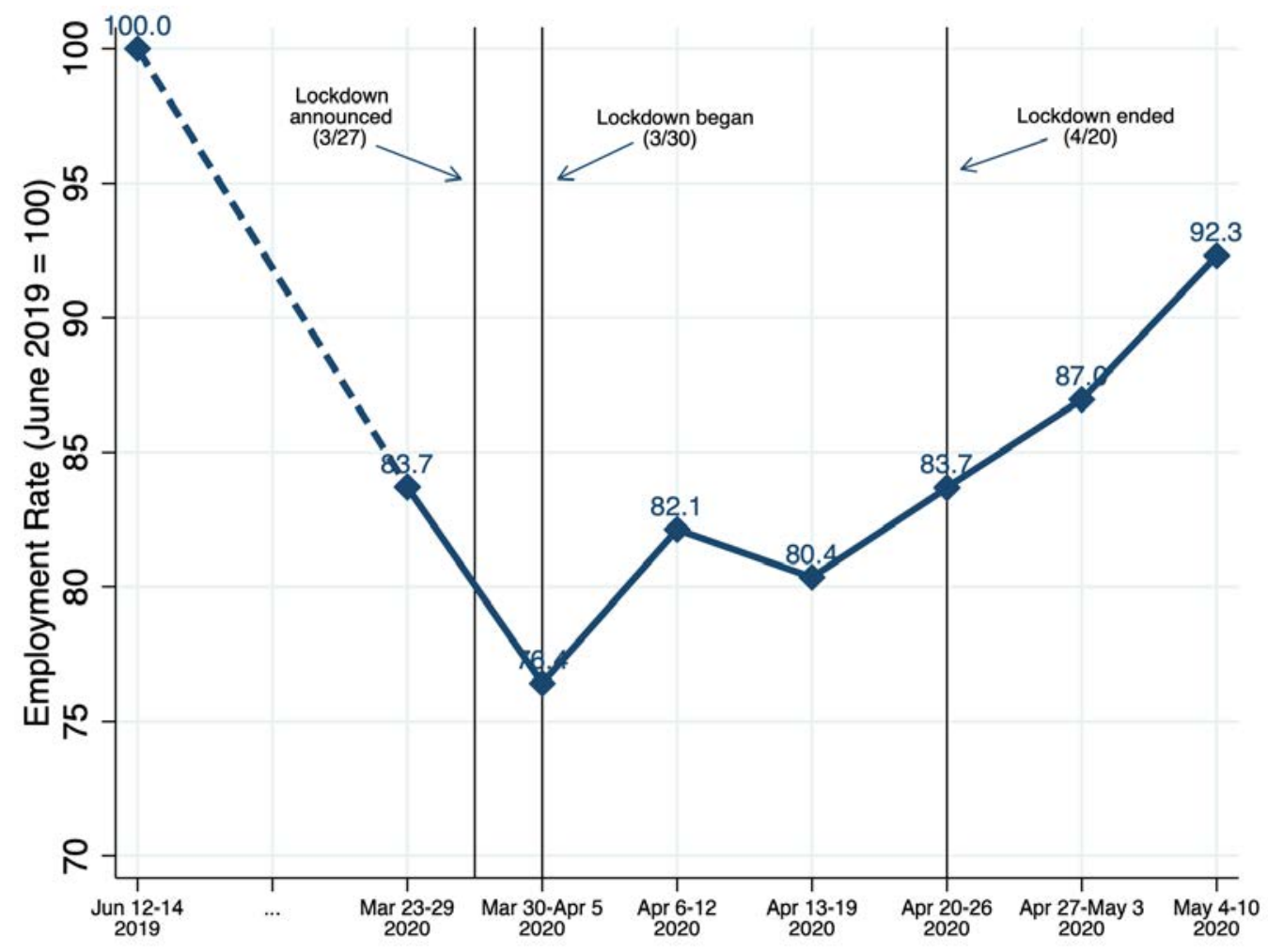

Note: This figure plots the employment in Accra and Kumasi, the two biggest cities in Ghana, proxied by Google survey data. The survey question was "In the last WEEK, how many hours did you work for pay or profit?". Employment rate is the proportion of people who reported they had worked in last week. The employment rate from the same survey conducted in June 2019 was normalized to one. Sample size was 266 in March 23-29, 342 for April 1-5, 281 for April 6-12, 302 for April 13-19, and 500 for the rest. 
Figure A.6: Hours Worked in Ghana Around the Lockdown Period

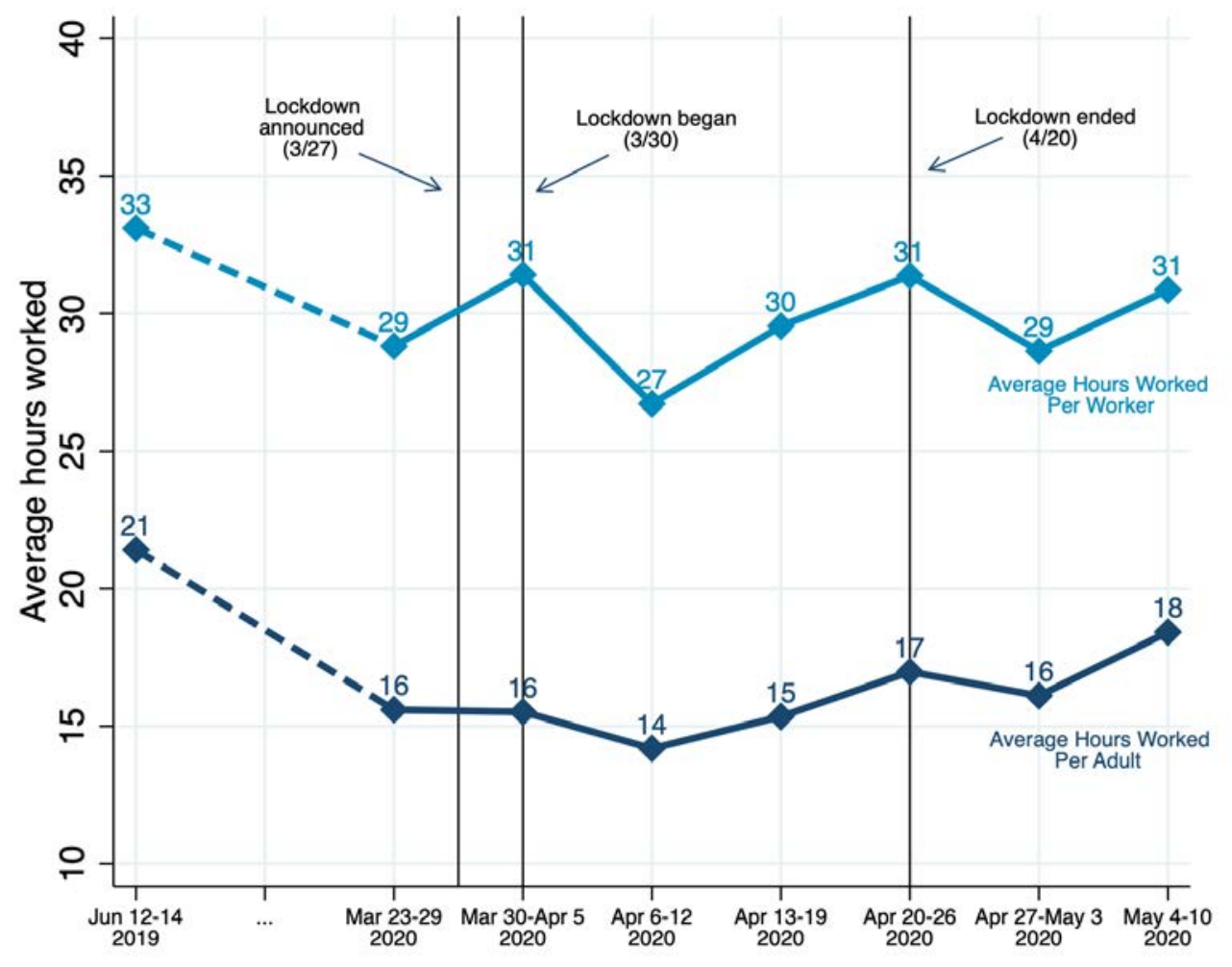

Note: This figure plots the employment in Accra and Kumasi, the two biggest cities in Ghana, proxied by Google survey data. The survey question was "In the last WEEK, how many hours did you work for pay or profit?". Average hours per worker is calculated by taking the average of hours among those who reported they had worked in the last week. Average hours per adult is calculated by taking the average of hours including those who reported they had not worked in the last week. Sample size was 266 in March 23-29, 342 for April 1-5, 281 for April 6-12, 302 for April 13-19, and 500 for the rest. 
Figure A.7: Simulated Deaths by Age under 36-week School Closure in Nigeria

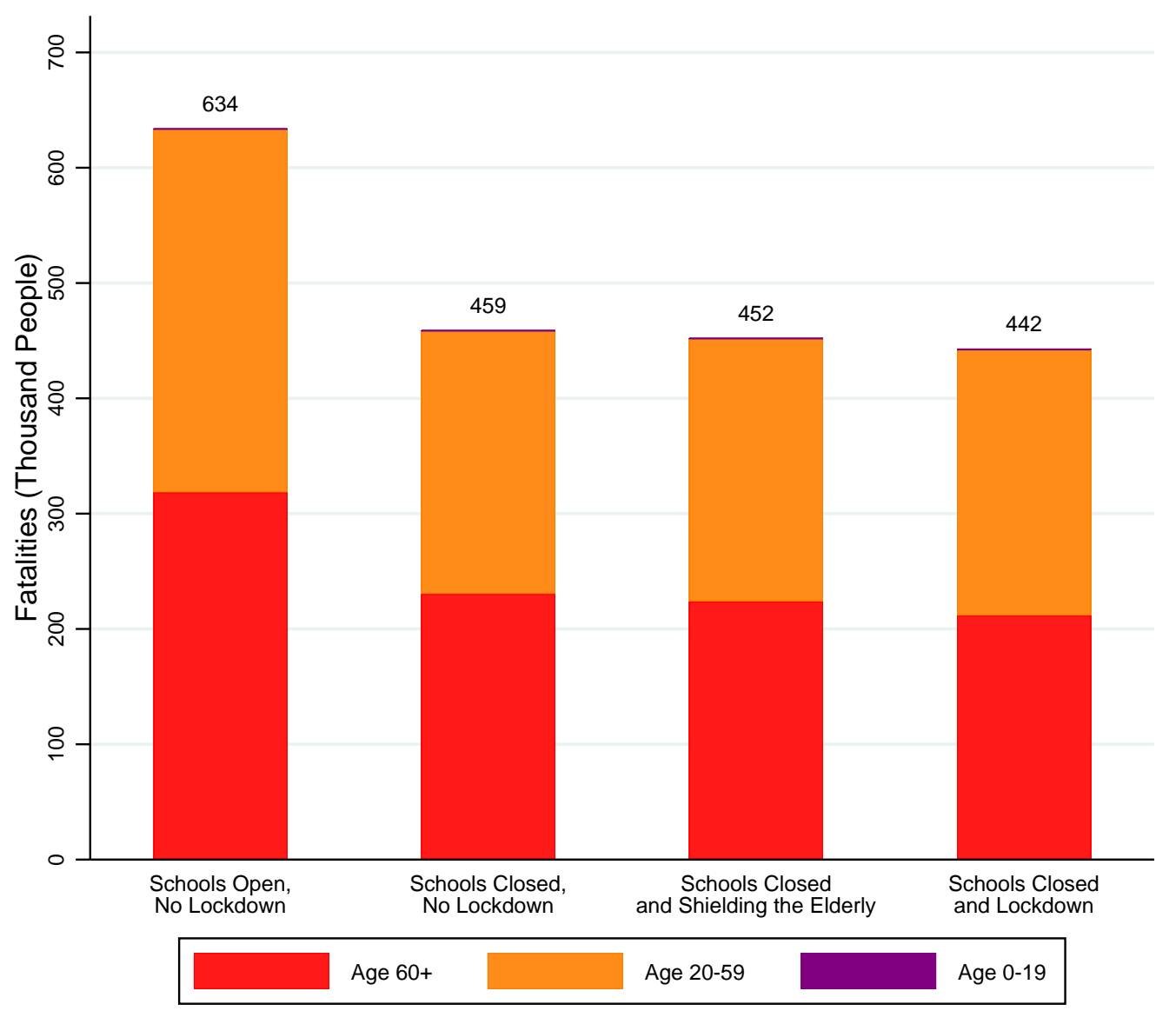

Note: This figure plots the number of deaths in total and by age group predicted by the model under various simulations about school closings and lockdown policies. 


\section{B. Appendix Tables}

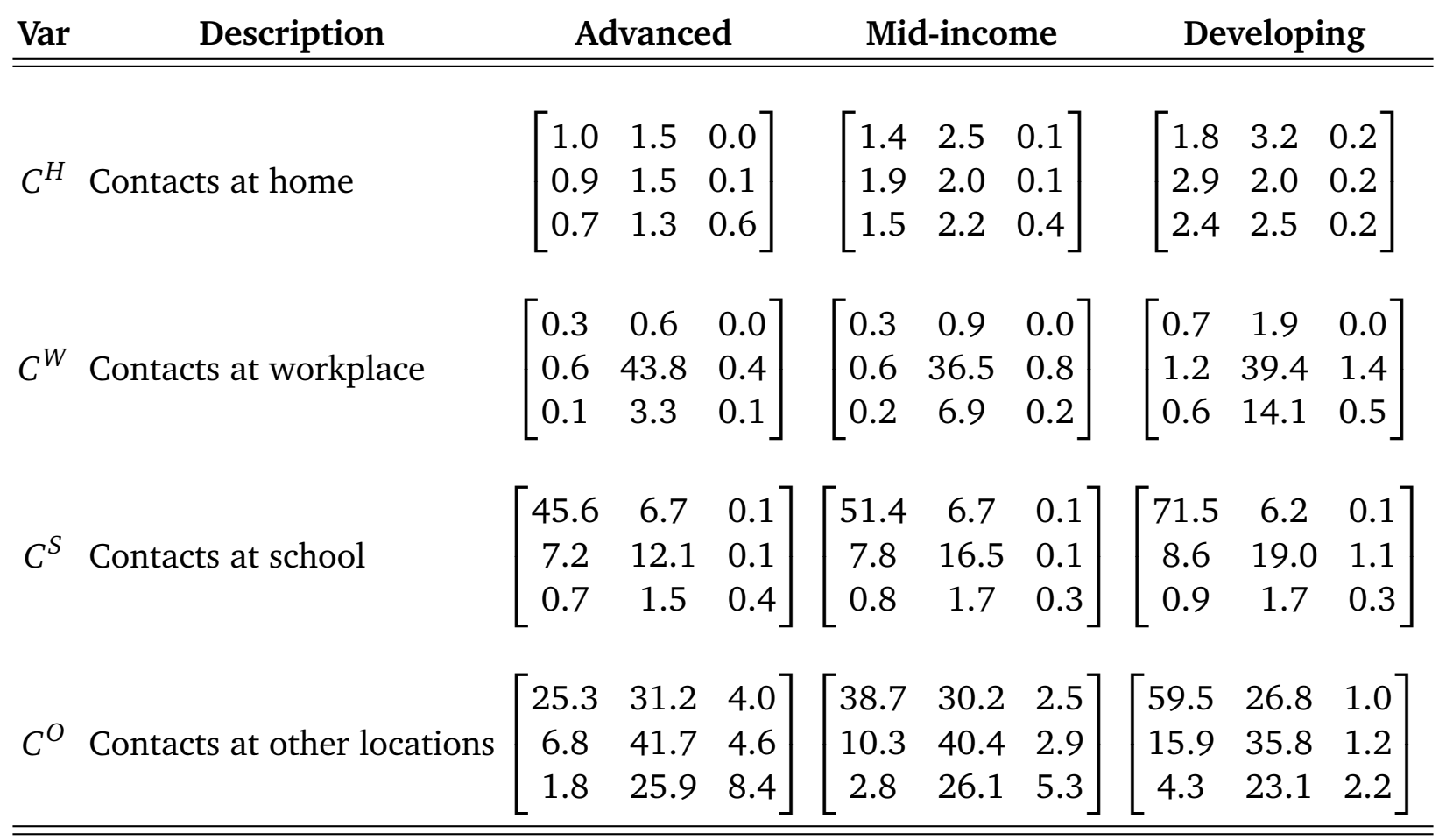

Table B.1: Contact Matrices 
Table B.2: ICU Bed Availability Across Countries

\begin{tabular}{lcc}
\hline \hline Country & $\begin{array}{c}\text { ICU beds per 100,000 } \\
\text { population }\end{array}$ & Per capita healthcare cost \\
\cline { 1 - 3 } \cline { 3 - 3 } United States & $20.0-31.7$ & $\$ 7,164$ \\
Canada & 13.5 & $\$ 3,867$ \\
Denmark & $6.7-8.9$ & $\$ 3,814$ \\
Australia & $8.0-8.9$ & $\$ 3,365$ \\
South Africa & 8.9 & $\$ 843$ \\
Sweden & $5.8-8.7$ & $\$ 3,622$ \\
Spain & $8.2-9.7$ & $\$ 2,941$ \\
Japan & 7.9 & $\$ 2,817$ \\
UK & $3.5-7.4$ & $\$ 3,222$ \\
New Zealand & $4.8-5.5$ & $\$ 2,655$ \\
China & $2.8-4.6$ & $\$ 265$ \\
Trinidad and Tobago & 2.1 & $\$ 1,237$ \\
Sri Lanka & 1.6 & $\$ 187$ \\
Zambia & 0 & $\$ 80$ \\
\hline \hline
\end{tabular}

Source: Table 1 in Prin and Wunsch (2012). Healthcare cost includes all public and private expenditures. 


\section{Contact Matrices for Each Containment Policy}

\section{C.1. Blanket lockdown}

We assume that children do not comply with the lockdown in 'other' location category. Therefore, there is no reduction in child-child contacts even under a blanket lockdown. The number of child-young and child-old contacts is still reduced under lockdowns because there are fewer yound and old adults. For simplicity, we assume that both contacts are reduced by the formal sector lockdown intensity.

Formal sector workers and children

$$
C=\underbrace{\left[\begin{array}{lll}
c_{c c}^{H} & c_{c y}^{H} & c_{c o}^{H} \\
c_{y c}^{H} & c_{y y}^{H} & c_{y o}^{H} \\
c_{o c}^{H} & c_{o y}^{H} & c_{o o}^{H}
\end{array}\right]}_{\text {Home contacts }}+\lambda \underbrace{\left[\begin{array}{ccc}
c_{c c}^{W} & c_{c y}^{W} & c_{c o}^{W} \\
c_{y c}^{W} & c_{y y}^{W} & c_{y o}^{W} \\
c_{o c}^{W} & c_{o y}^{W} & c_{o o}^{W}
\end{array}\right]}_{\text {Workplace contacts }}+\underbrace{\left[\begin{array}{ccc}
c_{c c}^{S} & c_{c y}^{S} & c_{c o}^{S} \\
c_{y c}^{S} & c_{y y}^{S} & c_{y o}^{S} \\
c_{o c}^{S} & c_{o y}^{S} & c_{o o}^{S}
\end{array}\right]}_{\text {School contacts }}+\underbrace{\left[\begin{array}{ccc}
c_{c c}^{O} & \lambda_{a} c_{c y}^{O} & \lambda_{a} c_{c o}^{O} \\
\lambda_{a} c_{y c}^{O} & \lambda_{a}^{2} c_{y y}^{O} & \lambda_{a}^{2} c_{y o}^{O} \\
\lambda_{a} c_{o c}^{O} & \lambda_{a}^{2} c_{o y}^{O} & \lambda_{a}^{2} c_{o o}^{O}
\end{array}\right]}_{\text {Other locations contacts }}
$$

\section{Informal sector worker}

$$
C=\underbrace{\left[\begin{array}{ccc}
c_{c c}^{H} & c_{c y}^{H} & c_{c o}^{H} \\
c_{y c}^{H} & c_{y y}^{H} & c_{y o}^{H} \\
c_{o c}^{H} & c_{o y}^{H} & c_{o o}^{H}
\end{array}\right]}_{\text {Home contacts }}+\underbrace{\left[\begin{array}{ccc}
c_{c c}^{W} & c_{c y}^{W} & c_{c o}^{W} \\
c_{y c}^{W} & c_{y y}^{W} & c_{y o}^{W} \\
c_{o c}^{W} & c_{o y}^{W} & c_{o o}^{W}
\end{array}\right]}_{\text {Workplace contacts }}+\underbrace{\left[\begin{array}{ccc}
c_{c c}^{S} & c_{c y}^{S} & c_{c o}^{S} \\
c_{y c}^{S} & c_{y y}^{S} & c_{y o}^{S} \\
c_{o c}^{S} & c_{o y}^{S} & c_{o o}^{S}
\end{array}\right]}_{\text {School contacts }}+\underbrace{\left[\begin{array}{ccc}
c_{c c}^{O} & c_{c y}^{O} & c_{c o}^{O} \\
c_{y c}^{O} & c_{y y}^{O} & c_{y o}^{O} \\
c_{o c}^{O} & c_{o y}^{O} & c_{o o}^{O}
\end{array}\right]}_{\text {Other locations contacts }}
$$

A blanket lockdown is equivalent to the combination of a young-specific and an old-specific lockdowns. In other words, if there is an age-specific lockdown applied to the young, an additional age-specific lockdown applied to the old should be equal to a blanket lockdown. This gives us the following relationship:

$$
1-\lambda_{a}^{2}=1-\lambda
$$

\section{C.2. Age-dependent lockdown}

Below are the contact matrices for age-dependent lockdown for old individuals. Other agedependent lockdowns targeting different age groups can be modelled in a similar way. 
Formal sector workers and children

$$
C=\underbrace{\left[\begin{array}{ccc}
c_{c c}^{H} & c_{c y}^{H} & c_{c o}^{H} \\
c_{y c}^{H} & c_{y y}^{H} & c_{y o}^{H} \\
c_{o c}^{H} & c_{o y}^{H} & c_{o o}^{H}
\end{array}\right]}_{\text {Home contacts }}+\underbrace{\left[\begin{array}{ccc}
c_{c c}^{W} & c_{c y}^{W} & \lambda_{a} c_{c o}^{W} \\
c_{y c}^{W} & c_{y y}^{W} & \lambda_{a} c_{y o}^{W} \\
\lambda_{a} c_{o c}^{W} & \lambda_{a} c_{o y}^{W} & \lambda_{a}^{2} c_{o o}^{W}
\end{array}\right]}_{\text {Workplace contacts }}+\underbrace{\left[\begin{array}{ccc}
c_{c c}^{S} & c_{c y}^{S} & c_{c o}^{S} \\
c_{y c}^{S} & c_{y y}^{S} & c_{y o}^{S} \\
c_{o c}^{S} & c_{o y}^{S} & c_{o o}^{S}
\end{array}\right]}_{\text {School contacts }}+\underbrace{\left[\begin{array}{ccc}
c_{c c}^{O} & c_{c y}^{O} & \lambda_{a} c_{c o}^{O} \\
c_{y c}^{O} & c_{y y}^{O} & \lambda_{a} c_{y o}^{O} \\
\lambda_{a} c_{o c}^{O} & \lambda_{a} c_{o y}^{O} & \lambda_{a}^{2} c_{o o}^{O}
\end{array}\right]}_{\text {Other locations contacts }}
$$

\section{Informal sector worker}

$$
C=\underbrace{\left[\begin{array}{ccc}
c_{c c}^{H} & c_{c y}^{H} & c_{c o}^{H} \\
c_{y c}^{H} & c_{y y}^{H} & c_{y o}^{H} \\
c_{o c}^{H} & c_{o y}^{H} & c_{o o}^{H}
\end{array}\right]}_{\text {Home contacts }}+\underbrace{\left[\begin{array}{ccc}
c_{c c}^{W} & c_{c y}^{W} & c_{c o}^{W} \\
c_{y c}^{W} & c_{y y}^{W} & c_{y o}^{W} \\
c_{o c}^{W} & c_{o y}^{W} & c_{o o}^{W}
\end{array}\right]}_{\text {Workplace contacts }}+\underbrace{\left[\begin{array}{ccc}
c_{c c}^{S} & c_{c y}^{S} & c_{c o}^{S} \\
c_{y c}^{S} & c_{y y}^{S} & c_{y o}^{S} \\
c_{o c}^{S} & c_{o y}^{S} & c_{o o}^{S}
\end{array}\right]}_{\text {School contacts }}+\underbrace{\left[\begin{array}{ccc}
c_{c c}^{O} & c_{c y}^{O} & c_{c o}^{O} \\
c_{y c}^{O} & c_{y y}^{O} & c_{y o}^{O} \\
c_{o c}^{O} & c_{o y}^{O} & c_{o o}^{O}
\end{array}\right]}_{\text {Other locations contacts }}
$$

\section{C.3. School Closure}

Let $\lambda_{s}$ be a policy parameter representing the degree of school closure, where $\lambda_{s}=0$ indicates full closure.

$$
C=\underbrace{\left[\begin{array}{ccc}
c_{c c}^{H} & c_{c y}^{H} & c_{c o}^{H} \\
c_{y c}^{H} & c_{y y}^{H} & c_{y o}^{H} \\
c_{o c}^{H} & c_{o y}^{H} & c_{o o}^{H}
\end{array}\right]}_{\text {Home contacts }}+\underbrace{\left[\begin{array}{ccc}
c_{c c}^{W} & c_{c y}^{W} & c_{c o}^{W} \\
c_{y c}^{W} & c_{y y}^{W} & c_{y o}^{W} \\
c_{o c}^{W} & c_{o y}^{W} & c_{o o}^{W}
\end{array}\right]}_{\text {Workplace contacts }}+\lambda_{s}^{\left[\begin{array}{ccc}
c_{c c}^{S} & c_{c y}^{S} & c_{c o}^{S} \\
c_{y c}^{S} & c_{y y}^{S} & c_{y o}^{S} \\
c_{o c}^{S} & c_{o y}^{S} & c_{o o}^{S}
\end{array}\right]}+\underbrace{\left[\begin{array}{ccc}
c_{c c}^{O} & c_{c y}^{O} & c_{c o}^{O} \\
c_{y c}^{O} & c_{y y}^{O} & c_{y o}^{O} \\
c_{o c}^{O} & c_{o y}^{O} & c_{o o}^{O}
\end{array}\right]}_{\text {School contacts }}
$$

